\title{
Entropy Solution for Doubly Nonlinear Elliptic Anisotropic Problems with Robin Boundary Conditions
}

\author{
I. Ibrango ${ }^{1}$ and S. Ouaro ${ }^{2}$ \\ ${ }^{1}$ Laboratoire de Mathématiques et Informatique (LAMI), UFR, Sciences et Techniques, Université Polytechnique de Bobo-Dioulasso, \\ 01 BP 1091, Bobo, Bobo-Dioulasso 01, Burkina Faso \\ ${ }^{2}$ Laboratoire de Mathématiques et Informatique (LAMI), UFR, Sciences Exactes et Appliquées, Université de Ouagadougou, \\ 03 BP 7021, Ouaga, Ouagadougou 03, Burkina Faso
}

Correspondence should be addressed to S. Ouaro; ouaro@yahoo.fr

Received 4 July 2015; Accepted 21 October 2015

Academic Editor: M. El-Gebeily

Copyright (C) 2015 I. Ibrango and S. Ouaro. This is an open access article distributed under the Creative Commons Attribution License, which permits unrestricted use, distribution, and reproduction in any medium, provided the original work is properly cited.

We study in this paper nonlinear anisotropic problems with Robin boundary conditions. We prove, by using the technic of monotone operators in Banach spaces, the existence of a sequence of weak solutions of approximation problems associated with the anisotropic Robin boundary value problem. For the existence and uniqueness of entropy solutions, we prove that the sequence of weak solutions converges to a measurable function which is the entropy solution of the anisotropic Robin boundary value problem.

\section{Introduction}

The aim of this paper is to study the following nonlinear anisotropic elliptic Robin boundary value problem:

$$
\begin{aligned}
-\sum_{i=1}^{N} \frac{\partial}{\partial x_{i}} a_{i}\left(x, \frac{\partial u}{\partial x_{i}}\right)+b(u) & =f \quad \text { in } \Omega, \\
\sum_{i=1}^{N} a_{i}\left(x, \frac{\partial u}{\partial x_{i}}\right) \eta_{i} & =-\gamma(u) \quad \text { on } \partial \Omega,
\end{aligned}
$$

where $\Omega$ is an open bounded domain of $\mathbb{R}^{N}(N \geq 3)$ with smooth boundary and meas $(\Omega)>0, f \in L^{1}(\Omega), \eta=$ $\left(\eta_{1}, \ldots, \eta_{N}\right)$ is the unit outward normal on $\partial \Omega$, and $\gamma(u)=$ $|u|^{r(x)-2} u$.
All papers on problems like (1) considered particular cases of function $b$. Indeed, in [1], Bonzi et al. studied the following problems:

$$
\begin{aligned}
-\sum_{i=1}^{N} \frac{\partial}{\partial x_{i}} a_{i}\left(x, \frac{\partial u}{\partial x_{i}}\right)+|u|^{p_{M}(x)-2} u & =f \quad \text { in } \Omega, \\
\sum_{i=1}^{N} a_{i}\left(x, \frac{\partial u}{\partial x_{i}}\right) \eta_{i} & =-|u|^{r(x)-2} u
\end{aligned}
$$

on $\partial \Omega$,

where $f \in L^{1}(\Omega)$. The authors use minimization technics used in [2] or [3] (see also $[4,5]$ ) to prove the existence and uniqueness of entropy solution.

The Robin type boundary conditions in the variable exponents setting are new and interesting problems and were for the first time studied by Boureanu and Radulescu in [3]. The main difficulty for the study of problem in [3] was the definition of an admissible space of solutions. The authors defined the appropriate space and obtained its properties 
which permit them to use minimization method to prove the existence of weak solutions to the following problem:

$$
\begin{aligned}
-\sum_{i=1}^{N} \frac{\partial}{\partial x_{i}} a_{i}\left(x, \frac{\partial u}{\partial x_{i}}\right)+b(x)|u|^{p_{M}(x)-2} u & =f(x, u) \\
& \text { in } \Omega, \\
u \geq 0 & \text { in } \Omega, \\
\sum_{i=1}^{N} a_{i}\left(x, \frac{\partial u}{\partial x_{i}}\right) \eta_{i}=g(x, u) & \text { on } \partial \Omega .
\end{aligned}
$$

Since we consider $L^{1}$-data $f$ instead of the function $f(x, u)$ considered in [3], the suitable notion of solution is the entropy solution introduced by Bénilan et al. in [6] (see also [7]). With the Robin type boundary conditions, the values of the solutions at the boundary must be precise and the notion of solutions considered must include the boundary condition. In this paper, as the function $b$ is more general, it is not possible to use minimization technic to get the existence of solution. Therefore, we used the technic of monotone operators in Banach spaces (see [8]) to get the existence of entropy solutions of (1).

For presenting our main result, we first have to describe the data involved in our problem. Let $\Omega$ be a bounded domain in $\mathbb{R}^{N}(N \geq 3)$ with smooth boundary domain $\partial \Omega$ and $\vec{p}(\cdot)=$ $\left(p_{1}(\cdot), \ldots, p_{N}(\cdot)\right)$ such that, for any $i=1, \ldots, N, p_{i}(\cdot): \bar{\Omega} \rightarrow$ $[2 ; N)$ is a continuous function with

$$
1<p_{i}^{-} \text {ess } \inf _{x \in \Omega} p_{i}(x) \leq \text { ess } \sup _{x \in \Omega} p_{i}(x) p_{i}^{+}<+\infty .
$$

For any $i=1, \ldots, N$, let $a_{i}: \Omega \times \mathbb{R} \rightarrow \mathbb{R}$ be a Carathéodory function satisfying the following:

(i) There exists a positive constant $C_{1}$ such that

$$
\left|a_{i}(x, \xi)\right| \leq C_{1}\left(j_{i}(x)+|\xi|^{p_{i}(x)-1}\right),
$$

for almost every $x \in \Omega$ and for every $\xi \in \mathbb{R}$, where $j_{i}$ is a nonnegative function lying in $L^{p_{i}^{\prime}(\cdot)}(\Omega)$, with $\left(1 / p_{i}(x)\right)+\left(1 / p_{i}^{\prime}(x)\right)=1$.

(ii) For $\xi, \eta \in \mathbb{R}$ with $\xi \neq \eta$ and for almost every $x \in \Omega$, there exists a positive constant $C_{2}$ such that

$$
\begin{aligned}
& \left(a_{i}(x, \xi)-a_{i}(x, \eta)\right)(\xi-\eta) \\
& \quad \geq \begin{cases}C_{2}|\xi-\eta|^{p_{i}(x)} & \text { if }|\xi-\eta| \geq 1, \\
C_{2}|\xi-\eta|^{p_{i}^{-}} & \text {if }|\xi-\eta|<1 .\end{cases}
\end{aligned}
$$

(iii) There exists a positive constant $C_{3}$ such that

$$
a_{i}(x, \xi) \cdot \xi \geq C_{3}|\xi|^{p_{i}(x)},
$$

for $\xi \in \mathbb{R}$ and for almost every $x \in \Omega$.
The hypotheses on $a_{i}$ are classical in the study of nonlinear problems (see $[1,3])$.

The function $b$ is such that

$b: \mathbb{R} \longrightarrow \mathbb{R}$ is continuous, surjective, nondecreasing

$$
\text { with } b(0)=0 \text {. }
$$

Throughout this paper, for any $i=1, \ldots, N$, we assume that

$$
\begin{aligned}
\frac{\bar{p}(N-1)}{N(\bar{p}-1)} & <p_{i}^{-}<\frac{\bar{p}(N-1)}{N-\bar{p}}, \\
\frac{p_{i}^{+}-p_{i}^{-}-1}{p_{i}^{-}} & <\frac{\bar{p}-N}{\bar{p}(N-1)}, \\
\sum_{i=1}^{N} \frac{1}{p_{i}^{-}} & >1,
\end{aligned}
$$

where $N / \bar{p}=\sum_{i=1}^{N}\left(1 / p_{i}^{-}\right)$.

We put for all $x \in \Omega$,

$$
\begin{aligned}
& p_{M}(x) \max \left\{p_{1}(x), \ldots, p_{N}(x)\right\}, \\
& p_{m}(x) \min \left\{p_{1}(x), \ldots, p_{N}(x)\right\}
\end{aligned}
$$

and for all $x \in \partial \Omega$,

$$
p^{\partial}(x)= \begin{cases}\frac{(N-1) p(x)}{N-p(x)} & \text { if } p(x)<N, \\ +\infty & \text { if } p(x) \geq N .\end{cases}
$$

We make the following assumption:

$$
r \in C(\bar{\Omega})
$$

$$
\text { with } 1<r^{-} \leq r^{+}<\min _{x \in \partial \Omega}\left\{p_{1}^{\partial}(x), \ldots, p_{N}^{\partial}(x)\right\} \text {. }
$$

Note that the function $\gamma$ is continuous, defined on $\mathbb{R}$ with $\gamma(t) t \geq 0$ for all $t$ in $\mathbb{R}$ and $\gamma(0)=0$.

A prototype example that is covered by our assumptions is the following anisotropic $\vec{p}(\cdot)$-harmonic system:

$$
-\sum_{i=1}^{N} \frac{\partial}{\partial x_{i}}\left(\left|\frac{\partial u}{\partial x_{i}}\right|^{p_{i}(x)-2} \frac{\partial u}{\partial x_{i}}\right)=f
$$

which, in the particular case when $p_{i}=p$ for any $i=1, \ldots, N$, is the $p$-Laplace equation.

The rest of the paper is organized as follows. In Section 2, we present some preliminary results. In Section 3, we study the existence and uniqueness of entropy solution.

\section{Preliminaries}

We recall in this section some definitions and basic properties of anisotropic Lebesgue and Sobolev spaces with variable exponent. Set

$$
C_{+}(\bar{\Omega})=\left\{p \in C(\bar{\Omega}) \text { such that } \min _{x \in \bar{\Omega}} p(x)>1\right\} .
$$


For any $p \in C_{+}(\bar{\Omega})$, the variable exponent Lebesgue space is defined by

$$
\begin{aligned}
& L^{p(\cdot)}(\Omega)\{u: \Omega \\
& \left.\quad \longrightarrow \mathbb{R} \text {, measurable such that } \int_{\Omega}|u|^{p(x)} d x<\infty\right\},
\end{aligned}
$$

endowed with the so-called Luxemburg norm

$$
\begin{aligned}
& |u|_{p(\cdot)} \\
& \quad \inf \left\{\lambda>0 \text { such that } \int_{\Omega}\left|\frac{u(x)}{\lambda}\right|^{p(x)} d x \leq 1\right\} .
\end{aligned}
$$

The $p(\cdot)$-modular of the $L^{p(\cdot)}(\Omega)$ space is the mapping $\rho_{p(\cdot)}$ : $L^{p(\cdot)}(\Omega) \rightarrow \mathbb{R}$ defined by

$$
\rho_{p(\cdot)}(u) \int_{\Omega}|u|^{p(x)} d x .
$$

For any $u \in L^{p(\cdot)}(\Omega)$, the following inequality (see $\left.[9,10]\right)$ will be used later:

$$
\begin{aligned}
\min \left\{|u|_{p(\cdot)}^{p^{-}} ;|u|_{p(\cdot)}^{p^{+}}\right\} & \leq \rho_{p(\cdot)}(u) \\
& \leq \max \left\{|u|_{p(\cdot)}^{p^{-}} ;|u|_{p(\cdot)}^{p^{+}}\right\} .
\end{aligned}
$$

For any $u \in L^{p(\cdot)}(\Omega)$ and $v \in L^{q(\cdot)}(\Omega)$ with $(1 / p(x))+$ $(1 / q(x))=1$ in $\Omega$, we have the following Hölder type inequality:

$$
\left|\int_{\Omega} u v d x\right| \leq\left(\frac{1}{p^{-}}+\frac{1}{q^{-}}\right)|u|_{p(\cdot)}|v|_{q(\cdot)} .
$$

If $\Omega$ is bounded and $p, q \in C_{+}(\bar{\Omega})$ such that $p(x) \leq q(x)$ for any $x \in \Omega$, then the embedding $L^{q(\cdot)}(\Omega) \hookrightarrow L^{p(\cdot)}(\Omega)$ is continuous (see [11, Theorem 2.8]).

Herein, we need the following anisotropic Sobolev space with variable exponent:

$$
\begin{aligned}
& W^{1, \vec{p}(\cdot)}(\Omega)\left\{u \in L^{p_{M}(\cdot)}(\Omega) \text { such that } \frac{\partial u}{\partial x_{i}}\right. \\
& \left.\quad \in L^{p_{i}(\cdot)}(\Omega), i=1, \ldots, N\right\} .
\end{aligned}
$$

$W^{1, \vec{p}(\cdot)}(\Omega)$ is a separable and reflexive Banach space (see [2]) under the norm

$$
\|u\|_{\vec{p}(\cdot)}=|u|_{p_{M}(\cdot)}+\sum_{i=1}^{N}\left|\frac{\partial u}{\partial x_{i}}\right|_{p_{i}(\cdot)} .
$$

We need the following embedding and trace results.

Theorem 1 (see [9, Corollary 2.1]). Let $\Omega \subset \mathbb{R}^{N}(N \geq 3)$ be a bounded open set and for all $i=1, \ldots, N, p_{i} \in L^{\infty}(\Omega)$, $p_{i}(x) \geq 1$ a.e. in $\Omega$. Then, for any $q \in L^{\infty}(\Omega)$ with $q(x) \geq 1$ a.e. in $\Omega$ such that

$$
\text { ess } \inf _{x \in \Omega}\left(p_{M}(x)-q(x)\right)>0,
$$

one has the compact embedding

$$
W^{1, \vec{p}(\cdot)}(\Omega) \hookrightarrow L^{q(\cdot)}(\Omega) .
$$

Theorem 2 (see [3, Theorem 6]). Let $\Omega \subset \mathbb{R}^{N}(N \geq 2)$ be a bounded open set with smooth boundary and let $\vec{p}(\cdot) \in$ $\left(C_{+}(\bar{\Omega})\right)^{N}, r \in C(\bar{\Omega})$ satisfy the condition

$$
1 \leq r(x)<\min \left\{p_{1}^{\partial}(x), \ldots, p_{N}^{\partial}(x)\right\}, \quad \forall x \in \partial \Omega .
$$

Then, there is a compact boundary trace embedding

$$
W^{1, \vec{p}(\cdot)}(\Omega) \hookrightarrow L^{r(\cdot)}(\partial \Omega) .
$$

We introduce the numbers

$$
\begin{aligned}
q & =\frac{N(\bar{p}-1)}{N-1}, \\
q^{*} & =\frac{N(\bar{p}-1)}{N-\bar{p}}=\frac{N q}{N-q} .
\end{aligned}
$$

The following result is due to Troisi (see [12]).

Theorem 3. Let $p_{1}, \ldots, p_{N} \in[1,+\infty) ; g \in W^{1,\left(p_{1}, \ldots, p_{N}\right)}(\Omega)$ and

$$
\begin{aligned}
& q=(\bar{p})^{*} \quad \text { if }(\bar{p})^{*}<N, \\
& q \in[1,+\infty) \quad \text { if }(\bar{p})^{*} \geq N .
\end{aligned}
$$

Then, there exists a constant $C_{4}>0$ depending on $N, p_{1}, \ldots$, $p_{N}$ if $\bar{p}<N$ and also on $q$ and meas $(\Omega)$ if $\bar{p} \geq N$ such that

$$
\|g\|_{L^{q}(\Omega)} \leq C_{4} \prod_{i=1}^{N}\left[\|g\|_{L^{p_{M}(\Omega)}}+\left\|\frac{\partial g}{\partial x_{i}}\right\|_{L^{p_{i}}(\Omega)}\right]^{1 / N} .
$$

In this paper, we will use the Marcinkiewicz space $\mathscr{M}^{q}(\Omega)$ $(1<q<+\infty)$ as the set of measurable functions $g: \Omega \rightarrow \mathbb{R}$ for which the distribution function

$$
\lambda_{g}(k)=\operatorname{meas}(\{x \in \Omega:|g(x)|>k\}), \quad k \geq 0
$$

satisfies an estimate of the form

$$
\lambda_{g}(k) \leq C k^{-q}, \quad \text { for some finite constant } C>0 .
$$

We will use the following pseudonorm in $\mathscr{M}^{q}(\Omega)$ :

$$
\|g\|_{\mathscr{M}^{q}(\Omega)} \inf \left\{C>0: \lambda_{g}(k) \leq C k^{-q}, \forall k>0\right\} .
$$

For any $k>0$, the truncation function $T_{k}$ is defined by

$$
T_{k}(s)=\max \{-k ; \min \{k ; s\}\} .
$$

It is clear that $\lim _{k \rightarrow+\infty} T_{k}(s)=s$ and $\left|T_{k}(s)\right|=\min \{|s| ; k\}$. 
In order to simplify the notation, for any $v \in W^{1, \vec{p}^{(\cdot)}}(\Omega)$, we use $v$ instead of $v_{l_{\partial \Omega}}$ for the trace of $v$ on $\partial \Omega$.

Set $\mathscr{T}^{1, \vec{p} \cdot()}(\Omega)$ as the set of the measurable functions $u$ : $\Omega \rightarrow \mathbb{R}$ such that, for any $k>0, T_{k}(u) \in W^{1, \vec{p}(\cdot)}(\Omega)$. We define the space $\mathscr{T}_{\operatorname{tr}}^{1, \vec{p}^{(\cdot)}}(\Omega)$ as the set of functions $u \in$ $\mathscr{T}^{1, \vec{p}(\cdot)}(\Omega)$ such that there exists a sequence $\left(u_{n}\right)_{n} \subset W^{1, \vec{p}(\cdot)}(\Omega)$ satisfying

$$
\begin{aligned}
& u_{n} \longrightarrow u \text { a.e. in } \Omega, \\
& \frac{\partial T_{k}\left(u_{n}\right)}{\partial x_{i}} \longrightarrow \frac{\partial T_{k}(u)}{\partial x_{i}} \quad \text { in } L^{1}(\Omega), \forall k>0,
\end{aligned}
$$

there exists a measurable function $v$ on $\partial \Omega$ such that $u_{n}$

$\longrightarrow v \quad$ a.e. on $\partial \Omega$.

We need the following lemma proved in [13].

Lemma 4. Let $g$ be a nonnegative function in $W^{1, \vec{p}(\cdot)}(\Omega)$. Assume $\bar{p}<N$ and there exists a constant $C>0$ such that

$$
\int_{\Omega}\left|T_{k}(g)\right|^{p_{M}^{-}} d x+\sum_{i=1}^{N} \int_{\{|g| \leq k\}}\left|\frac{\partial g}{\partial x_{i}}\right|^{p_{i}^{-}} d x \leq C(1+k)
$$

$\forall k>0$.

Then, there exists a constant $D$, depending on $C$, such that

$$
\|g\|_{M^{*} q^{*}(\Omega)} \leq D
$$

where $q^{*}=N(\bar{p}-1) /(N-\bar{p})$.

\section{Entropy Solutions}

The notion of entropy solutions to problem (1) where the data $f$ belongs to $L^{1}(\Omega)$ is the following.

Definition 5. A measurable function $u \in \mathscr{T}_{\operatorname{tr}}^{1, \vec{p}(\cdot)}(\Omega)$ is an entropy solution of problem (1) if $b(u) \in L^{1}(\Omega), \gamma(u) \in$ $L^{1}(\partial \Omega)$, and, for every $k>0$,

$$
\begin{aligned}
& \sum_{i=1}^{N} \int_{\Omega} a_{i}\left(x, \frac{\partial u}{\partial x_{i}}\right) \frac{\partial}{\partial x_{i}} T_{k}(u-\varphi) d x \\
& \quad+\int_{\Omega} b(u) T_{k}(u-\varphi) d x \\
& \quad+\int_{\partial \Omega} \gamma(u) T_{k}(u-\varphi) d \sigma \leq \int_{\Omega} f T_{k}(u-\varphi) d x
\end{aligned}
$$

for every $\varphi \in W^{1, \vec{p}(\cdot)}(\Omega) \cap L^{\infty}(\Omega)$.

The existence result is the following theorem.

Theorem 6. Assume that (4)-(12) hold. Then, problem (1) admits at least one entropy solution.
Proof. The proof is done in three steps.

Step 1 (the approximate problem). We define the reflexive space

$$
E=W^{1, \vec{p}(\cdot)}(\Omega) \times L^{p_{M}(\cdot)}(\partial \Omega)
$$

Let $X_{0}$ be the subspace of $E$ defined by

$$
X_{0}=\{(u, v) \in E: v=\tau(u)\}
$$

where $\tau(u)$ is the trace of $u \in \mathscr{T}_{\text {tr }}^{1, p(\cdot)}(\Omega)$ in the usual sense, since $u \in W^{1, \vec{p}(\cdot)}(\Omega)$. In the sequel, we will identify an element $(u, v) \in X_{0}$ with its representative $u \in W^{1, \vec{p}(\cdot)}(\Omega)$.

For any $n \in \mathbb{N}$ and $\varepsilon>0$, we consider the sequence of approximate problems:

$$
\begin{gathered}
\sum_{i=1}^{N} \int_{\Omega} a_{i}\left(x, \frac{\partial u_{n}}{\partial x_{i}}\right) \frac{\partial v}{\partial x_{i}} d x+\varepsilon \int_{\Omega}\left|u_{n}\right|^{p_{M}(x)-2} u_{n} v d x \\
\quad+\int_{\Omega} T_{n}\left(b\left(u_{n}\right)\right) v d x+\int_{\partial \Omega} T_{n}\left(\gamma\left(u_{n}\right)\right) v d \sigma \\
=\int_{\Omega} f_{n} v d x,
\end{gathered}
$$

where $f_{n}=T_{n}(f)$, and we define an operator $A_{n}$ by

$$
\begin{aligned}
\left\langle A_{n}(u), v\right\rangle= & \langle A(u), v\rangle+\int_{\Omega} T_{n}(b(u)) v d x \\
& +\int_{\partial \Omega} T_{n}(\gamma(u)) v d \sigma \quad \forall u, v \in X_{0},
\end{aligned}
$$

where

$$
\begin{aligned}
\langle A(u), v\rangle= & \sum_{i=1}^{N} \int_{\Omega} a_{i}\left(x, \frac{\partial u}{\partial x_{i}}\right) \frac{\partial v}{\partial x_{i}} d x \\
& +\varepsilon \int_{\Omega}|u|^{p_{M}(x)-2} u v d x
\end{aligned}
$$

Note that

$$
\begin{aligned}
&\left\|f_{n}\right\|_{\infty} \leq \frac{\|f\|_{1}}{\operatorname{meas}(\Omega)}, \\
&\left\|f_{n}\right\|_{1}=\int_{\Omega}\left|f_{n}\right| d x \leq \int_{\Omega}|f| d x=\|f\|_{1}, \\
& f_{n} \underset{n \rightarrow+\infty}{\longrightarrow} f \text { in } L^{1}(\Omega) \text {, a.e. in } \Omega .
\end{aligned}
$$


Assertion 1 (the operator $A$ is of type M). (i) The operator $A$ is monotone. Indeed, for $u, v \in W^{1, \vec{p}(\cdot)}(\Omega)$, we have

$$
\begin{aligned}
& \langle A(u)-A(v), u-v\rangle=\langle A(u), u-v\rangle+\langle A(v), v \\
& -u\rangle=\int_{\Omega} \sum_{i=1}^{N} a_{i}\left(x, \frac{\partial u}{\partial x_{i}}\right) \frac{\partial(u-v)}{\partial x_{i}} d x \\
& +\varepsilon \int_{\Omega}|u|^{p_{M}(x)-2} u(u-v) d x \\
& +\int_{\Omega} \sum_{i=1}^{N} a_{i}\left(x, \frac{\partial v}{\partial x_{i}}\right) \frac{\partial(v-u)}{\partial x_{i}} d x+\varepsilon \int_{\Omega}|v|^{p_{M}(x)-2} \\
& \cdot v(v-u) d x \\
& =\int_{\Omega} \sum_{i=1}^{N}\left[a_{i}\left(x, \frac{\partial u}{\partial x_{i}}\right)-a_{i}\left(x, \frac{\partial v}{\partial x_{i}}\right)\right] \\
& .\left(\frac{\partial u}{\partial x_{i}}-\frac{\partial v}{\partial x_{i}}\right) d x+\varepsilon \int_{\Omega}\left(|u|^{p_{M}(x)-2} u\right. \\
& \left.-|v|^{p_{M}(x)-2} v\right)(u-v) d x .
\end{aligned}
$$

Therefore,

$$
\langle A(u)-A(v), u-v\rangle \geq 0,
$$

since for $i=1, \ldots, N$, for almost every $x \in \Omega, a_{i}(x, \cdot)$ and $t \mapsto|t|^{p_{M}(x)-2} t$ are monotone.

(ii) The operator $A$ is hemicontinuous. Indeed, for every $u, v$ in $W^{1, \vec{p}(\cdot)}(\Omega)$, let

$$
\varphi: t \in \mathbb{R} \longmapsto \varphi(t)=\langle A(u+t v), v\rangle
$$

and let $t, t_{0} \in \mathbb{R}$ be such that $t \rightarrow t_{0}$. We have $w=u+t v \rightarrow$ $w_{0}=u+t_{0} v$ in $W^{1, \vec{p}(\cdot)}(\Omega)$.

Using the Hölder type inequality, there exists $i_{0} \in$ $\{1, \ldots, N\}$ such that

$$
\begin{aligned}
& \left|\varphi(t)-\varphi\left(t_{0}\right)\right|=\left|\langle A(u+t v), v\rangle-\left\langle A\left(u+t_{0} v\right), v\right\rangle\right| \\
& \quad \leq \sum_{i=1}^{N} \int_{\Omega}\left|a_{i}\left(x, \frac{\partial w}{\partial x_{i}}\right)-a_{i}\left(x, \frac{\partial w_{0}}{\partial x_{i}}\right)\right|\left|\frac{\partial v}{\partial x_{i}}\right| d x \\
& \quad+\left.\varepsilon \int_{\Omega}|| w\right|^{p_{M}(x)-2} w-\left|w_{0}\right|^{p_{M}(x)-2} w_{0}|| v \mid d x \\
& \quad \leq N\left(\frac{1}{p_{i_{0}}^{-}}+\frac{1}{\left(p_{i_{0}}^{\prime}\right)^{-}}\right) \\
& .\left|a_{i_{0}}\left(x, \frac{\partial w}{\partial x_{i_{0}}}\right)-a_{i_{0}}\left(x, \frac{\partial w_{0}}{\partial x_{i_{0}}}\right)\right|_{p_{i_{0}}^{\prime}(\cdot)}\left|\frac{\partial v}{\partial x_{i_{0}}}\right|_{p_{i_{0}}(\cdot)} \\
& \quad+\varepsilon\left(\frac{1}{p_{M}^{-}}+\frac{1}{\left(p_{M}^{\prime}\right)^{-}}\right) \\
& .\left.|| w\right|^{p_{M}(x)-2} w-\left.\left|w_{0}\right|^{p_{M}(x)-2} w_{0}\right|_{p_{M}^{\prime}(\cdot)}|v|_{p_{M}(\cdot)} .
\end{aligned}
$$

Let us denote $\psi_{i_{0}}(x, w)=a_{i_{0}}\left(x, \partial w / \partial x_{i_{0}}\right)$.
Using assumption (5) and [11, Theorems 4.1 and 4.2] we have $\psi_{i_{0}}(x, w) \rightarrow \psi_{i_{0}}\left(x, w_{0}\right)$ in $L^{p_{i_{0}}^{\prime}(\cdot)}(\Omega)$. Then, we deduce that $\varphi$ is continuous; namely, the operator $A$ is hemicontinuous.

Since the operator $A$ is monotone and hemicontinuous, then according to Lemma 2.1 in [8], $A$ is of type $\mathrm{M}$.

Assertion 2 (the operator $A_{n}$ is of type M). Indeed, let $\left(u_{k}\right)_{k \in \mathbb{N}}$ be a sequence in $X_{0}$ such that

$$
\begin{aligned}
u_{k} & \rightarrow u \quad \text { weakly in } X_{0}, \\
A_{n} u_{k} & \rightarrow \chi \quad \text { weakly in } X_{0}^{\prime}, \\
\lim _{k \rightarrow+\infty} \sup _{k \rightarrow+\infty}\left\langle A_{n}\left(u_{k}\right), u_{k}\right\rangle & \leq\langle\chi, u\rangle .
\end{aligned}
$$

Since

$$
\begin{aligned}
& T_{n}(b(u)) u \geq 0, \\
& T_{n}(\gamma(u)) u \geq 0,
\end{aligned}
$$

by Fatou's lemma, we obtain that

$$
\begin{gathered}
\liminf _{k \rightarrow+\infty}\left(\int_{\Omega} T_{n}\left(b\left(u_{k}\right)\right) u_{k} d x+\int_{\partial \Omega} T_{n}\left(\gamma\left(u_{k}\right)\right) u_{k} d \sigma\right) \\
\geq \int_{\Omega} T_{n}(b(u)) u d x+\int_{\partial \Omega} T_{n}(\gamma(u)) u d \sigma
\end{gathered}
$$

and thanks to the Lebesgue dominated convergence theorem, we have

$$
\begin{gathered}
\lim _{k \rightarrow+\infty}\left(\int_{\Omega} T_{n}\left(b\left(u_{k}\right)\right) v d x+\int_{\partial \Omega} T_{n}\left(\gamma\left(u_{k}\right)\right) v d \sigma\right) \\
=\int_{\Omega} T_{n}(b(u)) v d x+\int_{\partial \Omega} T_{n}(\gamma(u)) v d \sigma,
\end{gathered}
$$

for all $v$ in $X_{0}$. Consequently,

$$
\begin{array}{r}
T_{n}\left(b\left(u_{k}\right)\right)+T_{n}\left(\gamma\left(u_{k}\right)\right) \rightarrow T_{n}(b(u))+T_{n}(\gamma(u)) \\
\text { weakly in } X_{0}^{\prime} .
\end{array}
$$

Therefore, we deduce that

$$
A u_{k} \rightarrow \chi-\left(T_{n}(b(u))+T_{n}(\gamma(u))\right) \text { weakly in } X_{0}^{\prime} .
$$

As in Assertion 1, we prove that the operator $A$ is of type M, so we have

$$
A u=\chi-\left(T_{n}(b(u))+T_{n}(\gamma(u))\right) .
$$

Thus, it follows that

$$
A_{n} u=\chi
$$

Hence, $A_{n}$ is of type $\mathrm{M}$.

Assertion 3 (the operator $A_{n}$ is coercive). Indeed, since

$$
T_{n}(b(u)) u+T_{n}(\gamma(u)) u \geq 0,
$$


then

$$
\left\langle A_{n}(u), u\right\rangle \geq\langle A(u), u\rangle .
$$

According to (7), we have

$$
\langle A(u), u\rangle \geq C_{3} \int_{\Omega} \sum_{i=1}^{N}\left|\frac{\partial u}{\partial x_{i}}\right|^{p_{i}(x)} d x+\varepsilon \int_{\Omega}|u|^{p_{M}(x)} d x
$$

Denote

$$
\begin{aligned}
& \mathscr{I}=\left\{i \in\{1, \ldots, N\}:\left|\frac{\partial u}{\partial x_{i}}\right|_{p_{i}(\cdot)} \leq 1\right\}, \\
& \mathscr{J}=\left\{i \in\{1, \ldots, N\}:\left|\frac{\partial u}{\partial x_{i}}\right|_{p_{i}(\cdot)}>1\right\} .
\end{aligned}
$$

We have

$$
\begin{aligned}
\int_{\Omega} \sum_{i=1}^{N}\left|\frac{\partial u}{\partial x_{i}}\right|^{p_{i}(x)} d x= & \sum_{i \in \mathcal{F}} \int_{\Omega}\left|\frac{\partial u}{\partial x_{i}}\right|^{p_{i}(x)} d x \\
& +\sum_{i \in \mathscr{J}} \int_{\Omega}\left|\frac{\partial u}{\partial x_{i}}\right|^{p_{i}(x)} d x \\
\geq & \sum_{i \in \mathscr{F}}\left|\frac{\partial u}{\partial x_{i}}\right|_{p_{i}(\cdot)}^{p_{i}^{+}}+\sum_{i \in \mathscr{J}}\left|\frac{\partial u}{\partial x_{i}}\right|_{p_{i}(\cdot)}^{p_{i}^{-}} \\
\geq & \sum_{i \in \mathcal{F}}\left|\frac{\partial u}{\partial x_{i}}\right|_{p_{i}(\cdot)}^{p_{i}^{-}} \geq \sum_{i \in \mathcal{J}}\left|\frac{\partial u}{\partial x_{i}}\right|_{p_{i}(\cdot)}^{p_{m}^{-}} \\
\geq & \sum_{i=1}^{N}\left|\frac{\partial u}{\partial x_{i}}\right|_{p_{i}(\cdot)}^{p_{m}^{-}}-\sum_{i \in \mathscr{F}}\left|\frac{\partial u}{\partial x_{i}}\right|_{p_{i}(\cdot)}^{p_{m}^{-}} \\
\geq & \sum_{i=1}^{N}\left|\frac{\partial u}{\partial x_{i}}\right|_{p_{i}(\cdot)}^{p_{m}^{-}}-N .
\end{aligned}
$$

Using the convexity of the application $t \in \mathbb{R}^{+} \mapsto t^{p_{m}^{-}}, p_{m}^{-}>1$, we obtain

$$
\int_{\Omega} \sum_{i=1}^{N}\left|\frac{\partial u}{\partial x_{i}}\right|^{p_{i}(x)} d x \geq \frac{1}{N^{p_{m}^{-}-1}}\left(\sum_{i=1}^{N}\left|\frac{\partial u}{\partial x_{i}}\right|_{p_{i}(\cdot)}\right)^{p_{m}^{-}}-N .
$$

Then,

$$
\begin{aligned}
\left\langle A_{n}(u), u\right\rangle \geq & \frac{C_{3}}{N^{p_{m}^{-}-1}}\left(\sum_{i=1}^{N}\left|\frac{\partial u}{\partial x_{i}}\right|_{p_{i}(\cdot)}\right)^{p_{m}^{-}} \\
& +\varepsilon \int_{\Omega}|u|^{p_{M}(x)} d x-C_{3} N .
\end{aligned}
$$

(i) Assume $|u|_{p_{M}(\cdot)}>1$. Then, (18) gives $\int_{\Omega}|u|^{p_{M}(x)} d x \geq$ $|u|_{p_{M}(\cdot)}^{p_{m}^{-}}$
So, combining (56) and (61) we get

$$
\begin{array}{r}
\left\langle A_{n}(u), u\right\rangle \geq C\left[\left(\sum_{i=1}^{N}\left|\frac{\partial u}{\partial x_{i}}\right|_{p_{i}(\cdot)}\right)^{p_{m}^{-}}+|u|_{p_{M}(\cdot)}^{p_{m}^{-}}\right] \\
-C_{3} N \geq \frac{C}{2^{p_{m}^{-}-1}}\|u\|_{\vec{p}(\cdot)}^{p_{m}^{-}}-C_{3} N, \\
\text { where } C=\min \left\{\frac{C_{3}}{N^{p_{m}^{-}-1}} ; \varepsilon\right\} .
\end{array}
$$
we get

(ii) Assume $|u|_{p_{M}(\cdot)} \leq 1$. Then, combining (56) and (61)

$$
\begin{aligned}
&\left\langle A_{n}(u), u\right\rangle \geq C\left[\left(\sum_{i=1}^{N}\left|\frac{\partial u}{\partial x_{i}}\right|_{p_{i}(\cdot)}\right)^{p_{m}^{-}}+|u|_{p_{M}(\cdot)}^{p_{m}^{-}}\right]-1 \\
&-C_{3} N+\varepsilon \int_{\Omega}|u|^{p_{M}(x)} d x \\
& \geq \frac{C}{2^{p_{m}^{-}-1}}\|u\|_{\vec{p}(\cdot)}^{p_{m}^{-}}-1-C_{3} N, \\
& \text { where } C=\min \left\{\frac{C_{3}}{N^{p_{m}^{-}-1}} ; 1\right\} .
\end{aligned}
$$

Consequently, since $p_{m}^{-}>1$, the operator $A_{n}$ is coercive.

Besides, the operator $A_{n}$ is bounded and hemicontinuous.

Then, for any $F_{n}=T_{n}(f) \in E^{\prime} \subset X_{0}^{\prime}$, we can deduce the existence of a function $u_{n} \in X_{0}$ such that

$$
\left\langle A_{n}\left(u_{n}\right), v\right\rangle=\left\langle F_{n}, v\right\rangle, \quad \forall v \in X_{0} .
$$

Namely, $u_{n}$ is a weak solution of problem (39).

We are now going to prove that these approximated solutions $u_{n}$ tend, as $n$ goes to infinity, to a measurable function $u$ which is an entropy solution of problem (1). To start with, we establish some a priori estimates.

Step 2 (a priori estimates). Assume (4)-(12) and let $u_{n}$ be a solution of problem (39). We have the following results.

Lemma 7. There exists a constant $C_{5}>0$ such that

$$
\begin{aligned}
& \int_{\Omega}\left|T_{k}\left(u_{n}\right)\right|^{p_{M}^{-}} d x+\sum_{i=1}^{N} \int_{\left\{\left|u_{n}\right| \leq k\right\}}\left|\frac{\partial u_{n}}{\partial x_{i}}\right|^{p_{i}^{-}} d x \\
& \leq C_{5}(k+1) .
\end{aligned}
$$

Proof. Let us take $T_{k}\left(u_{n}\right)$ as a test function in (39). Since

$$
\begin{gathered}
\int_{\Omega} T_{n}\left(b\left(u_{n}\right)\right) T_{k}\left(u_{n}\right) d x+\varepsilon \int_{\Omega}\left|u_{n}\right|^{p_{M}(x)-2} u_{n} T_{k}\left(u_{n}\right) \\
+\int_{\partial \Omega} T_{n}\left(\gamma\left(u_{n}\right)\right) T_{k}\left(u_{n}\right) d \sigma \geq 0,
\end{gathered}
$$


using relation (7), we obtain

$$
C_{3} \sum_{i=1}^{N} \int_{\left\{\left|u_{n}\right| \leq k\right\}}\left|\frac{\partial u_{n}}{\partial x_{i}}\right|^{p_{i}(x)} d x \leq k\|f\|_{1} .
$$

Then, we have

$$
\begin{aligned}
& \sum_{i=1}^{N} \int_{\left\{\left|u_{n}\right| \leq k\right\}}\left|\frac{\partial u_{n}}{\partial x_{i}}\right|^{p_{i}^{-}} d x \\
& =\sum_{i=1}^{N} \int_{\left\{\left|u_{n}\right| \leq k ;\left|\partial u_{n} / \partial x_{i}\right|>1\right\}}\left|\frac{\partial u_{n}}{\partial x_{i}}\right|^{p_{i}^{-}} d x \\
& \quad+\sum_{i=1}^{N} \int_{\left\{\left|u_{n}\right| \leq k ;\left|\partial u_{n} / \partial x_{i}\right| \leq 1\right\}}\left|\frac{\partial u_{n}}{\partial x_{i}}\right|^{p_{i}^{-}} d x \\
& \leq \sum_{i=1}^{N} \int_{\left\{\left|u_{n}\right| \leq k\right\}}\left|\frac{\partial u_{n}}{\partial x_{i}}\right|^{p_{i}(x)} d x+N \cdot \operatorname{meas}(\Omega) \\
& \leq \frac{k}{C_{3}}\|f\|_{1}+N \cdot \operatorname{meas}(\Omega) .
\end{aligned}
$$

Moreover, we have

$$
\begin{aligned}
\int_{\Omega}\left|T_{k}\left(u_{n}\right)\right|^{p_{M}^{-}} d x= & \int_{\left\{\left|T_{k}\left(u_{n}\right)\right| \leq 1\right\}}\left|T_{k}\left(u_{n}\right)\right|^{p_{M}^{-}} d x \\
& +\int_{\left\{\left|T_{k}\left(u_{n}\right)\right|>1\right\}}\left|T_{k}\left(u_{n}\right)\right|^{p_{M}^{-}} d x \\
\leq & \operatorname{meas}(\Omega)+\int_{\left\{\left|T_{k}\left(u_{n}\right)\right|>1\right\}} k^{p_{M}^{-}} d x \\
\leq & \operatorname{meas}(\Omega)\left(1+k^{p_{M}^{-}}\right) .
\end{aligned}
$$

Therefore, we get

$$
\begin{aligned}
& \int_{\Omega}\left|T_{k}\left(u_{n}\right)\right|^{p_{M}^{-}} d x+\sum_{i=1}^{N} \int_{\left\{\left|u_{n}\right| \leq k\right\}}\left|\frac{\partial u_{n}}{\partial x_{i}}\right|^{p_{i}^{-}} d x \\
& \leq \operatorname{meas}(\Omega)\left(1+N+k^{p_{M}^{-}}\right)+\frac{k}{C_{3}}\|f\|_{1} \\
& \leq C_{5}(1+k)
\end{aligned}
$$

where $C_{5}=\max \left\{\operatorname{meas}(\Omega)\left(1+N+k^{p_{M}^{-}}\right) ;\left(1 / C_{3}\right)\|f\|_{1}\right\}$.

Lemma 8. For any $k>0$, there exist two constants $C_{7}>0$ and $C_{8}>0$ such that

(i) $\left\|u_{n}\right\|_{\mathscr{M}^{q^{*}(\Omega)}} \leq C_{7}$,

(ii) $\left\|\partial u_{n} / \partial x_{i}\right\|_{\mathscr{M}^{p^{-} q / \bar{p}}(\Omega)} \leq C_{8}, \forall i=1, \ldots, N$.
Proof. (i) This is a consequence of Lemmas 4 and 7.

(ii)

(a) Let $\alpha \geq 1$. For any $k \geq 1$, we have

$$
\begin{aligned}
\lambda_{\partial u_{n} / \partial x_{i}}(\alpha)= & \operatorname{meas}\left(\left\{x \in \Omega:\left|\frac{\partial u_{n}}{\partial x_{i}}\right|>\alpha\right\}\right) \\
= & \operatorname{meas}\left(\left\{\left|\frac{\partial u_{n}}{\partial x_{i}}\right|>\alpha ;\left|u_{n}\right| \leq k\right\}\right) \\
& +\operatorname{meas}\left(\left\{\left|\frac{\partial u_{n}}{\partial x_{i}}\right|>\alpha ;\left|u_{n}\right|>k\right\}\right) \\
\leq & \int_{\left\{\left|\partial u_{n} / \partial x_{i}\right|>\alpha ;\left|u_{n}\right| \leq k\right\}} d x+\lambda_{u_{n}}(k) \\
\leq & \int_{\left\{\left|u_{n}\right| \leq k\right\}}\left(\frac{1}{\alpha}\left|\frac{\partial u_{n}}{\partial x_{i}}\right|\right)^{p_{i}^{-}} d x+\lambda_{u_{n}}(k) \\
\leq & \alpha^{-p_{i}^{-}} C^{\prime} k+C k^{-q^{*}} .
\end{aligned}
$$

Then, there exists a positive constant $C_{6}$ such that

$$
\lambda_{\partial u_{n} / \partial x_{i}}(\alpha) \leq C_{6}\left(k \alpha^{-p_{i}^{-}}+k^{-q^{*}}\right) .
$$

Let us consider the function

$$
\begin{aligned}
g:[1,+\infty) & \longrightarrow \mathbb{R}, \\
t & \longmapsto g(t)=\frac{t}{\alpha^{p_{i}^{-}}}+t^{-q^{*}} .
\end{aligned}
$$

We have $g^{\prime}(t)=0$ for $t=\left(q^{*} \alpha^{p_{i}^{-}}\right)^{1 /\left(q^{*}+1\right)}$. Thus, if we take $k=\left(q^{*} \alpha^{p_{i}^{-}}\right)^{1 /\left(q^{*}+1\right)} \geq 1$ in (72) we get

$$
\begin{aligned}
\lambda_{\partial u_{n} / \partial x_{i}}(\alpha) & \leq C_{6} k\left(\frac{q^{*}+1}{q^{*}} \frac{1}{\alpha^{p_{i}^{-}}}\right) \leq C_{6}^{\prime} \alpha^{-\left(q^{*} /\left(q^{*}+1\right)\right) p_{i}^{-}} \\
& \leq C_{6}^{\prime} \alpha^{-p_{i}^{-} q / \bar{p}}
\end{aligned}
$$

$\forall \alpha \geq 1$, where $C_{6}^{\prime}$ is a positive constant.

(b) If $0 \leq \alpha<1$, we have

$$
\begin{aligned}
\lambda_{\partial u_{n} / \partial x_{i}}(\alpha) & =\operatorname{meas}\left(\left\{\left|\frac{\partial u_{n}}{\partial x_{i}}\right|>\alpha\right\}\right) \leq \operatorname{meas}(\Omega) \\
& \leq \operatorname{meas}(\Omega) \alpha^{-p_{i}^{-} q / \bar{p}} .
\end{aligned}
$$

Then

$$
\lambda_{\partial u_{n} / \partial x_{i}}(\alpha) \leq\left(C_{6}^{\prime}+\operatorname{meas}(\Omega)\right) \alpha^{-p_{i}^{-} q / \bar{p}}, \quad \forall \alpha \geq 0 .
$$

Therefore, we deduce that there exists a positive constant $C_{8}$ such that

$$
\left\|\frac{\partial u_{n}}{\partial x_{i}}\right\|_{\mathscr{M}^{p_{i}^{-} q / \bar{p}}(\Omega)} \leq C_{8}, \quad \forall i=1, \ldots, N .
$$


Step 3 (existence of entropy solution). Using Lemma 8, we have the following useful lemma (see [13]).

Lemma 9. For $i=1, \ldots, N$, as $n \rightarrow+\infty$, one has

$$
a_{i}\left(x, \frac{\partial u_{n}}{\partial x_{i}}\right) \longrightarrow a_{i}\left(x, \frac{\partial u}{\partial x_{i}}\right) \text { in } L^{1}(\Omega) \text { a.e. } x \in \Omega \text {. }
$$

In order to pass to the limit in relation (39), we also need the following convergence results which can be proved as in [7] (see also [1, 13]).

Proposition 10. Assume (4)-(12). If $u_{n} \in W^{1, \vec{p}(\cdot)}(\Omega)$ is a weak solution of $\left(P_{n}\right)$ then the sequence $\left(u_{n}\right)_{n \in \mathbb{N}^{*}}$ is Cauchy in measure. In particular, there exist a measurable function $u$ and a subsequence still denoted by $u_{n}$ such that $u_{n} \rightarrow u$ in measure.

Proposition 11. Assume (4)-(12). If $u_{n} \in W^{1, \vec{p}(\cdot)}(\Omega)$ is a weak solution of $\left(P_{n}\right)$ then

(i) there exists $s>1$ such that $u_{n} \rightarrow u$ a.e. in $\Omega$ and moreover $u_{n} \rightarrow u$ in $W^{1, s}(\Omega)$,

(ii) for all $i=1, \ldots, N, \partial u_{n} / \partial x_{i}$ converges strongly in $L^{1}(\Omega)$. Moreover $a_{i}\left(x, \partial u_{n} / \partial x_{i}\right)$ converges to $a_{i}\left(x, \partial u / \partial x_{i}\right)$ in $L^{1}(\Omega)$ strongly and in $L^{p_{i}^{\prime}(\cdot)}(\Omega)$ weakly for all $i=1, \ldots, N$,

(iii) $u_{n}$ converges to some measurable function $v$ a.e. in $\partial \Omega$.

We can now pass to the limit in relation (39).

Let $\varphi \in W^{1, \vec{p}(\cdot)}(\Omega) \cap L^{\infty}(\Omega)$ and choosing $T_{k}\left(u_{n}-\varphi\right)$ as a test function in (39), we get

$$
\begin{aligned}
& \sum_{i=1}^{N} \int_{\Omega} a_{i}\left(x, \frac{\partial u_{n}}{\partial x_{i}}\right) \frac{\partial}{\partial x_{i}} T_{k}\left(u_{n}-\varphi\right) d x \\
& \quad+\int_{\Omega} T_{n}\left(b\left(u_{n}\right)\right) T_{k}\left(u_{n}-\varphi\right) d x \\
& \quad+\varepsilon \int_{\Omega}\left|u_{n}\right|^{p_{M}(x)-2} u_{n} T_{k}\left(u_{n}-\varphi\right) d x \\
& \quad+\int_{\partial \Omega} T_{n}\left(\gamma\left(u_{n}\right)\right) T_{k}\left(u_{n}-\varphi\right) d \sigma \\
& =\int_{\Omega} f_{n} T_{k}\left(u_{n}-\varphi\right) d x .
\end{aligned}
$$

For the right-hand side of (79), we have

$$
\int_{\Omega} f_{n}(x) T_{k}\left(u_{n}-\varphi\right) d x \longrightarrow \int_{\Omega} f(x) T_{k}(u-\varphi) d x
$$

since $f_{n}$ converges strongly to $f$ in $L^{1}(\Omega)$ and $T_{k}\left(u_{n}-\varphi\right)$ converges weakly- ${ }^{*}$ to $T_{k}(u-\varphi)$ in $L^{\infty}(\Omega)$ and a.e. in $\Omega$.

For the first term of (79), we have (see [13])

$$
\begin{gathered}
\liminf _{n \rightarrow+\infty} \sum_{i=1}^{N} \int_{\Omega} a_{i}\left(x, \frac{\partial u_{n}}{\partial x_{i}}\right) \frac{\partial}{\partial x_{i}} T_{k}\left(u_{n}-\varphi\right) d x \\
\geq \sum_{i=1}^{N} \int_{\Omega} a_{i}\left(x, \frac{\partial u}{\partial x_{i}}\right) \frac{\partial}{\partial x_{i}} T_{k}(u-\varphi) d x .
\end{gathered}
$$

We now focus our attention on the second term of (79). We have

$$
\begin{aligned}
& T_{n}\left(b\left(u_{n}\right)\right) T_{k}\left(u_{n}-\varphi\right) \longrightarrow b(u) T_{k}(u-\varphi) \\
& \text { a.e. } x \in \Omega, \\
& \left|T_{n}\left(b\left(u_{n}\right)\right) T_{k}\left(u_{n}-\varphi\right)\right| \leq k\left|b\left(u_{n}\right)\right| .
\end{aligned}
$$

Now we show that $\left|b\left(u_{n}\right)\right| \leq\|f\|_{1} /$ meas $(\Omega)$. Indeed, let us denote by

$$
\begin{array}{r}
H_{\delta}(s)=\min \left(\frac{s^{+}}{\delta} ; 1\right), \\
\operatorname{sign}_{0}^{+}(s)= \begin{cases}1 & \text { if } s>0 \\
0 & \text { if } s \leq 0\end{cases}
\end{array}
$$

If $\theta$ is a maximal monotone operator defined on $\mathbb{R}$, we denote by $\theta_{0}$ the main section of $\theta$; that is,

$$
\begin{aligned}
& \theta_{0}(s) \\
& = \begin{cases}\text { minimal absolute value of } \theta(s) & \text { if } \theta(s) \neq \emptyset, \\
+\infty & \text { if }[s,+\infty) \cap D(\theta)=\emptyset, \\
-\infty & \text { if }(-\infty, s] \cap D(\theta)=\emptyset .\end{cases}
\end{aligned}
$$

Remark that as $\delta$ goes to $0, H_{\delta}(s)$ goes to $\operatorname{sign}_{0}^{+}(s)$.

We take $\varphi=H_{\delta}\left(u_{n}-M\right)$ as a test function in (39) for the weak solution $u_{n}$ and $M>0$ (a constant to be chosen later) to get

$$
\begin{aligned}
& \sum_{i=1}^{N} \int_{\Omega} a_{i}\left(x, \frac{\partial u_{n}}{\partial x_{i}}\right) \frac{\partial}{\partial x_{i}} H_{\delta}\left(u_{n}-M\right) d x \\
& \quad+\varepsilon \int_{\Omega}\left|u_{n}\right|^{p_{M}(x)-2} u_{n} H_{\delta}\left(u_{n}-M\right) d x \\
& \quad+\int_{\Omega} T_{n}\left(b\left(u_{n}\right)\right) H_{\delta}\left(u_{n}-M\right) d x \\
& \quad+\int_{\partial \Omega} T_{n}\left(\gamma\left(u_{n}\right)\right) H_{\delta}\left(u_{n}-M\right) d \sigma \\
& =\int_{\Omega} f_{n} H_{\delta}\left(u_{n}-M\right) d x .
\end{aligned}
$$


We have

$$
\begin{aligned}
& \sum_{i=1}^{N} \int_{\Omega} a_{i}\left(x, \frac{\partial u_{n}}{\partial x_{i}}\right) \frac{\partial}{\partial x_{i}} H_{\delta}\left(u_{n}-M\right) d x \\
& =\frac{1}{\delta} \sum_{i=1}^{N} \int_{\left\{\left(u_{n}-M\right)^{+} / \delta<1\right\}} a_{i}\left(x, \frac{\partial u_{n}}{\partial x_{i}}\right) \frac{\partial}{\partial x_{i}}\left(u_{n}-M\right)^{+} d x \\
& =\frac{1}{\delta} \sum_{i=1}^{N} \int_{\left\{0<u_{n}-M<\delta\right\}} a_{i}\left(x, \frac{\partial u_{n}}{\partial x_{i}}\right) \frac{\partial}{\partial x_{i}} u_{n} d x \\
& \geq 0 \text { according to }(7), \\
& \int_{\Omega}\left|u_{n}\right|^{p_{M}(x)-2} u_{n} H_{\delta}\left(u_{n}-M\right) d x \\
& =\int_{\left\{\left(u_{n}-M\right)^{+} / \delta<1\right\}}\left|u_{n}\right|^{p_{M}(x)-2} u_{n} \frac{\left(u_{n}-M\right)^{+}}{\delta} d x \\
& \quad+\int_{\left\{\left(u_{n}-M\right)^{+} / \delta \geq 1\right\}}\left|u_{n}\right|^{p_{M}(x)-2} u_{n} d x \\
& \geq \frac{1}{\delta} \int_{\left\{M<u_{n}<M+\delta\right\}}\left|u_{n}\right|^{p_{M}(x)-2} u_{n}\left(u_{n}-M\right) d x \geq 0 .
\end{aligned}
$$

Note also that since the function $\gamma$ is nondecreasing with $\gamma(0)=0$, we have

$$
\begin{aligned}
\int_{\partial \Omega} & T_{n}\left(\gamma\left(u_{n}\right)\right) H_{\delta}\left(u_{n}-M\right) d \sigma \\
= & \int_{\partial \Omega \cap\left\{\left(u_{n}-M\right)^{+} / \delta<1\right\}} T_{n}\left(\gamma\left(u_{n}\right)\right) H_{\delta}\left(u_{n}-M\right) d \sigma \\
& +\int_{\partial \Omega \cap\left\{\left(u_{n}-M\right)^{+} / \delta \geq 1\right\}} T_{n}\left(\gamma\left(u_{n}\right)\right) H_{\delta}\left(u_{n}-M\right) d \sigma \\
= & \int_{\partial \Omega \cap\left\{\left(u_{n}-M\right)^{+} / \delta<1\right\}} T_{n}\left(\gamma\left(u_{n}\right)\right) \frac{\left(u_{n}-M\right)^{+}}{\delta} d \sigma \\
& +\int_{\partial \Omega \cap\left\{\left(u_{n}-M\right)^{+} / \delta \geq 1\right\}} T_{n}\left(\gamma\left(u_{n}\right)\right) d \sigma \\
= & \frac{1}{\delta} \int_{\partial \Omega \cap\left\{0<u_{n}-M<\delta\right\}} T_{n}\left(\gamma\left(u_{n}\right)\right)\left(u_{n}-M\right) d \sigma \\
& +\int_{\partial \Omega \cap\left\{u_{n}-M \geq \delta\right\}} T_{n}\left(\gamma\left(u_{n}\right)\right) d \sigma \geq 0 .
\end{aligned}
$$

Then, (85) gives

$$
\begin{gathered}
\int_{\Omega} T_{n}\left(b\left(u_{n}\right)\right) H_{\delta}\left(u_{n}-M\right) d x \\
\leq \int_{\Omega} f_{n} H_{\delta}\left(u_{n}-M\right) d x,
\end{gathered}
$$

which is equivalent to saying

$$
\begin{gathered}
\int_{\Omega}\left(T_{n}\left(b\left(u_{n}\right)\right)-T_{n}(b(M))\right) H_{\delta}\left(u_{n}-M\right) d x \\
\leq \int_{\Omega}\left(f_{n}-T_{n}(b(M))\right) H_{\delta}\left(u_{n}-M\right) d x .
\end{gathered}
$$

We now let $\delta$ go to 0 in the above inequality to obtain

$$
\begin{aligned}
& \int_{\Omega}\left(T_{n}\left(b\left(u_{n}\right)\right)-T_{n}(b(M))\right)^{+} d x \\
& \quad \leq \int_{\Omega}\left(f_{n}-T_{n}(b(M))\right) \operatorname{sign}_{0}^{+}\left(u_{n}-M\right) d x .
\end{aligned}
$$

Choosing $M=b_{0}^{-1}\left(\left\|f_{n}\right\|_{\infty}\right)$ in the above inequality (since $b$ is surjective), we obtain

$$
\begin{aligned}
& \int_{\Omega}\left(T_{n}\left(b\left(u_{n}\right)\right)-T_{n}\left(\left\|f_{n}\right\|_{\infty}\right)\right)^{+} d x \\
& \leq \int_{\Omega}\left(f_{n}-T_{n}\left(\left\|f_{n}\right\|_{\infty}\right)\right) \\
& \cdot \operatorname{sign}_{0}^{+}\left(u_{n}-b_{0}^{-1}\left(\left\|f_{n}\right\|_{\infty}\right)\right) d x .
\end{aligned}
$$

For any $n>\|f\|_{1} /$ meas $(\Omega)$, we have

$$
\begin{aligned}
& \int_{\Omega}\left(f_{n}-T_{n}\left(\left\|f_{n}\right\|_{\infty}\right)\right) \operatorname{sign}_{0}^{+}\left(u_{n}-b_{0}^{-1}\left(\left\|f_{n}\right\|_{\infty}\right)\right) d x \\
& \quad=\int_{\Omega}\left(f_{n}-\left\|f_{n}\right\|_{\infty}\right) \operatorname{sign}_{0}^{+}\left(u_{n}-b_{0}^{-1}\left(\left\|f_{n}\right\|_{\infty}\right)\right) d x \\
& \quad \leq 0 .
\end{aligned}
$$

Then, (91) gives

$$
\int_{\Omega}\left(T_{n}\left(b\left(u_{n}\right)\right)-\left\|f_{n}\right\|_{\infty}\right)^{+} d x \leq 0, \quad \forall n>\frac{\|f\|_{1}}{\operatorname{meas}(\Omega)} .
$$

Hence, for all $n>\|f\|_{1} / \operatorname{meas}(\Omega)$, we have $\left(T_{n}\left(b\left(u_{n}\right)\right)-\right.$ $\left.\left\|f_{n}\right\|_{\infty}\right)^{+}=0$ a.e. in $\Omega$, which implies that

$$
T_{n}\left(b\left(u_{n}\right)\right) \leq\left\|f_{n}\right\|_{\infty} \quad \forall n>\frac{\|f\|_{1}}{\operatorname{meas}(\Omega)} .
$$


Let us remark that as $u_{n}$ is a weak solution of (39), then $\left(-u_{n}\right)$ is a weak solution to the following problem:

$$
\left(\widetilde{P}_{n}\right) \begin{cases}-\sum_{i=1}^{N} \frac{\partial}{\partial x_{i}} \widetilde{a}_{i}\left(x, \frac{\partial u_{n}}{\partial x_{i}}\right)+T_{n}\left(\widetilde{b}\left(u_{n}\right)\right)+\varepsilon\left|u_{n}\right|^{p_{M}(x)-2} u_{n}=\widetilde{f}_{n} & \text { in } \Omega, \\ \sum_{i=1}^{N} \widetilde{a}_{i}\left(x, \frac{\partial u_{n}}{\partial x_{i}}\right) \eta_{i}=-\gamma\left(u_{n}\right) & \text { on } \partial \Omega,\end{cases}
$$

where $\widetilde{a}_{i}(x, \xi)=-a_{i}(x,-\xi), \widetilde{b}(s)=-b(-s)$, and $\widetilde{f}_{n}=-f_{n}$.

According to (94) we deduce that

$$
T_{n}\left(-b\left(u_{n}\right)\right) \leq\left\|f_{n}\right\|_{\infty} \quad \forall n>\frac{\|f\|_{1}}{\operatorname{meas}(\Omega)} .
$$

Therefore

$$
T_{n}\left(b\left(u_{n}\right)\right) \geq-\left\|f_{n}\right\|_{\infty} \quad \forall n>\frac{\|f\|_{1}}{\operatorname{meas}(\Omega)} .
$$

It follows from (94) and (97) that, for all $n>\|f\|_{1} /$ meas $(\Omega)$, $\left|T_{n}\left(b\left(u_{n}\right)\right)\right| \leq\left\|f_{n}\right\|_{\infty}$ which implies

$$
\left|b\left(u_{n}\right)\right| \leq\left\|f_{n}\right\|_{\infty} \leq \frac{\|f\|_{1}}{\operatorname{meas}(\Omega)} \quad \text { a.e. in } \Omega \text {. }
$$

We can now use the Lebesgue dominated convergence theorem to get

$$
\begin{gathered}
\lim _{n \rightarrow+\infty} \int_{\Omega} T_{n}\left(b\left(u_{n}\right)\right) T_{k}\left(u_{n}-\varphi\right) d x \\
=\int_{\Omega} b(u) T_{k}(u-\varphi) d x .
\end{gathered}
$$

By using again the Lebesgue dominated convergence theorem, we obtain

$$
\begin{gathered}
\int_{\partial \Omega} T_{n}\left(\gamma\left(u_{n}\right)\right) T_{k}\left(u_{n}-\varphi\right) d \sigma \\
\longrightarrow \int_{\partial \Omega} \gamma(u) T_{k}(u-\varphi) d \sigma .
\end{gathered}
$$

For the third term of (79), let us prove that

$$
\liminf _{n \rightarrow+\infty} \varepsilon \int_{\Omega}\left|u_{n}\right|^{p_{M}(x)-2} u_{n} T_{k}\left(u_{n}-\varphi\right) d x \geq 0
$$

$$
\text { for } \varepsilon \longrightarrow 0 \text {. }
$$

We have

$$
\begin{aligned}
& \int_{\Omega}\left|u_{n}\right|^{p_{M}(x)-2} u_{n} T_{k}\left(u_{n}-\varphi\right) d x \\
& =\int_{\Omega}\left(\left|u_{n}\right|^{p_{M}(x)-2} u_{n}-|\varphi|^{p_{M}(x)-2} \varphi\right) T_{k}\left(u_{n}-\varphi\right) d x \\
& \quad+\int_{\Omega}|\varphi|^{p_{M}(x)-2} \varphi T_{k}\left(u_{n}-\varphi\right) d x .
\end{aligned}
$$

Since the quantity $\left(\left|u_{n}\right|^{p_{M}(x)-2} u_{n}-|\varphi|^{p_{M}(x)-2} \varphi\right) T_{k}\left(u_{n}-\varphi\right)$ is nonnegative and since for all $x$ in $\Omega$, the application $\xi \mapsto$ $|\xi|^{p_{M}(x)-2} \xi$ is continuous, we have

$$
\begin{array}{r}
\left(\left|u_{n}\right|^{p_{M}(x)-2} u_{n}-|\varphi|^{p_{M}(x)-2} \varphi\right) T_{k}\left(u_{n}-\varphi\right) \\
\longrightarrow\left(|u|^{p_{M}(x)-2} u-|\varphi|^{p_{M}(x)-2} \varphi\right) T_{k}(u-\varphi) \\
\text { a.e. in } \Omega
\end{array}
$$

and by Fatou's lemma, it follows that

$$
\begin{gathered}
\liminf _{n \rightarrow+\infty} \int_{\Omega}\left(\left|u_{n}\right|^{p_{M}(x)-2} u_{n}-|\varphi|^{p_{M}(x)-2} \varphi\right) \\
\cdot T_{k}\left(u_{n}-\varphi\right) d x \geq \int_{\Omega}\left(|u|^{p_{M}(x)-2} u\right. \\
\left.-|\varphi|^{p_{M}(x)-2} \varphi\right) T_{k}(u-\varphi) d x .
\end{gathered}
$$

We have

$$
\begin{aligned}
\left.\int_{\Omega}|| \varphi\right|^{p_{M}(x)-2} \varphi \mid d x= & \int_{\Omega}|\varphi|^{p_{M}(x)-1} \varphi d x \\
\leq & \int_{\Omega}\left(\|\varphi\|_{\infty}\right)^{p_{M}(x)-1} d x \\
\leq & \int_{\left\{\|\varphi\|_{\infty} \leq 1\right\}}\left(\|\varphi\|_{\infty}\right)^{p_{M}(x)-1} d x \\
& +\int_{\left\{\|\varphi\|_{\infty}>1\right\}}\left(\|\varphi\|_{\infty}\right)^{p_{M}(x)-1} d x \\
\leq & \operatorname{meas}(\Omega) \\
& +\left(\|\varphi\|_{\infty}\right)^{p_{M}^{+}-1} \text { meas }(\Omega) \\
< & +\infty .
\end{aligned}
$$

Hence, $|\varphi|^{p_{M}(x)-2} \varphi \in L^{1}(\Omega)$.

Since $T_{k}\left(u_{n}-\varphi\right)$ converges weakly- ${ }^{*}$ to $T_{k}(u-\varphi)$ in $L^{\infty}(\Omega)$ and $|\varphi|^{p_{M}(x)-2} \varphi \in L^{1}(\Omega)$, it follows that

$$
\begin{gathered}
\lim _{n \rightarrow+\infty} \int_{\Omega}|\varphi|^{p_{M}(x)-2} \varphi T_{k}\left(u_{n}-\varphi\right) d x \\
=\int_{\Omega}|\varphi|^{p_{M}(x)-2} \varphi T_{k}(u-\varphi) d x .
\end{gathered}
$$


By adding (104) and (106), we get

$$
\begin{gathered}
\liminf _{n \rightarrow+\infty} \int_{\Omega}\left|u_{n}\right|^{p_{M}(x)-2} u_{n} T_{k}\left(u_{n}-\varphi\right) d x \\
\geq \int_{\Omega}|u|^{p_{M}(x)-2} u T_{k}(u-\varphi) d x .
\end{gathered}
$$

Since

$$
\begin{aligned}
& \int_{\Omega}|u|^{p_{M}(x)-2} u T_{k}(u-\varphi) d x \leq k \int_{\Omega}|u|^{p_{M}(x)-1} d x \\
& \quad<+\infty
\end{aligned}
$$

thus we get

$$
\begin{aligned}
\liminf _{n \rightarrow+\infty} \varepsilon \int_{\Omega}\left|u_{n}\right|^{p_{M}(x)-2} u_{n} T_{k}\left(u_{n}-\varphi\right) d x & \geq 0 \\
& \text { for } \varepsilon \longrightarrow 0 .
\end{aligned}
$$

Combining (80), (81), (99), (100), and (109) we obtain

$$
\begin{aligned}
\sum_{i=1}^{N} \int_{\Omega} & a_{i}\left(x, \frac{\partial u}{\partial x_{i}}\right) \frac{\partial}{\partial x_{i}} T_{k}(u-\varphi) d x \\
& +\int_{\Omega} b(u) T_{k}(u-\varphi) d x \\
& +\int_{\partial \Omega} \gamma(u) T_{k}(u-\varphi) d \sigma \leq \int_{\Omega} f T_{k}(u-\varphi) d x .
\end{aligned}
$$

Then, $u$ is an entropy solution of problem (1). That completes the proof of Theorem 6 .

We now state the uniqueness result of entropy solution.

Theorem 12. Assume that (4)-(12) hold true and let $u$ be an entropy solution of (1). Then, $u$ is unique.

Proof. The proof is done in two steps.

Step 1 (a priori estimates). We consider the following.

Lemma 13. Assume (4)-(12) and $f \in L^{1}(\Omega)$. Let $u$ be an entropy solution of (1). Then

$$
\sum_{i=1}^{N} \int_{\{|u| \leq k\}}\left|\frac{\partial u}{\partial x_{i}}\right|^{p_{i}(x)} d x \leq \frac{k}{C_{3}}\|f\|_{1}
$$

and there exists a positive constant $C_{9}$ such that

$$
\|b(u)\|_{1} \leq C_{9} \cdot \operatorname{meas}(\Omega)+\|f\|_{1} .
$$

Proof. Let us take $\varphi=0$ in the entropy inequality (36).

(i) By the fact that $\int_{\Omega} b(u) T_{k}(u) d x+\int_{\partial \Omega} \gamma(u) T_{k}(u) d \sigma \geq$ 0 , using (7), we get (111).

(ii) Also, using the fact that $\sum_{i=1}^{N} \int_{\Omega} a_{i}\left(x, \partial u / \partial x_{i}\right)(\partial /$ $\left.\partial x_{i}\right) T_{k}(u) d x+\int_{\partial \Omega} \gamma(u) T_{k}(u) d \sigma \geq 0$, relation (36) gives

$$
\int_{\Omega} b(u) T_{k}(u) d x \leq \int_{\Omega} f(x) T_{k}(u) d x .
$$

By (113), we deduce that

$$
\begin{aligned}
& \int_{\{|u| \leq k\}} b(u) T_{k}(u) d x+\int_{\{|u|>k\}} b(u) T_{k}(u) d x \\
& \quad \leq k\|f\|_{1}
\end{aligned}
$$

which imply that

$$
\int_{\{|u|>k\}} b(u) T_{k}(u) d x \leq k\|f\|_{1}
$$

or

$$
\int_{\{u>k\}} b(u) d x+\int_{\{u<-k\}}-b(u) d x \leq\|f\|_{1} .
$$

Therefore,

$$
\int_{\{|u|>k\}}|b(u)| d x \leq\|f\|_{1}
$$

So, we obtain

$$
\begin{aligned}
\int_{\Omega}|b(u)| d x & =\int_{\{|u| \leq k\}}|b(u)| d x+\int_{\{|u|>k\}}|b(u)| d x \\
& \leq \int_{\{|u| \leq k\}}|b(u)| d x+\|f\|_{1} .
\end{aligned}
$$

Since the function $b$ is nondecreasing, then

$$
\int_{\{|u| \leq k\}}|b(u)| d x \leq \max \{b(k) ;|b(-k)|\} \cdot \operatorname{meas}(\Omega) .
$$

Consequently, there exists a constant $C_{9}=\max \{b(k) ;|b(-k)|\}$ such that

$$
\|b(u)\|_{1} \leq C_{9} \cdot \operatorname{meas}(\Omega)+\|f\|_{1} .
$$

Lemma 14. Assume (4)-(12) and let $f \in L^{1}(\Omega)$. If $u$ is an entropy solution of (1), then there exists a constant $D$ which depends on $f$ and $\Omega$ such that

$$
\operatorname{meas}(\{|u|>k\}) \leq \frac{D}{\min \{b(k),|b(-k)|\}}, \quad \forall k>0
$$

and a constant $D^{\prime}>0$ which depends on $f$ and $\Omega$ such that

$$
\operatorname{meas}\left(\left\{\left|\frac{\partial u}{\partial x_{i}}\right|>k\right\}\right) \leq \frac{D^{\prime}}{k^{1 /\left(p_{M}^{-}\right)^{\prime}}}, \quad \forall k \geq 1 .
$$

Proof. (i) For any $k>0$, relation (112) gives

$$
\begin{aligned}
& \int_{\{|u|>k\}} \min \{b(k),|b(-k)|\} d x \leq \int_{\{|u|>k\}}|b(u)| d x \\
& \leq C_{9} \cdot \operatorname{meas}(\Omega)+\|f\|_{1} .
\end{aligned}
$$

Therefore,

$$
\begin{gathered}
\min \{b(k),|b(-k)|\} \cdot \operatorname{meas}(\{|u|>k\}) \\
\leq C_{9} \cdot \operatorname{meas}(\Omega)+\|f\|_{1}=D ;
\end{gathered}
$$


that is,

$$
\operatorname{meas}(\{|u|>k\}) \leq \frac{D}{\min \{b(k),|b(-k)|\}} .
$$

(ii) See [7] for the poof of (122).

Lemma 15. Assume (4)-(12) and let $f \in L^{1}(\Omega)$. If $u$ is an entropy solution of (1), then

$$
\lim _{h \rightarrow+\infty} \int_{\Omega}|f| \chi_{\{|u|>h-t\}} d x=0
$$

where $h>0$ and $t>0$.

Proof. Since the function $b$ is surjective, according to Lemma 14-(121), we have

$$
\lim _{h \rightarrow+\infty} \operatorname{meas}(\{|u|>h-t\})=0
$$

and as $f \in L^{1}(\Omega)$, it follows by using the Lebesgue dominated convergence theorem that

$$
\lim _{h \rightarrow+\infty} \int_{\Omega}|f| \chi_{\{|u|>h-t\}} d x=0
$$

Lemma 16. Assume (4)-(12) and let $f \in L^{1}(\Omega)$. If $u$ is an entropy solution of (1), then there exists a positive constant $K$ such that

$$
\rho_{p_{i}^{\prime}(\cdot)}\left(\left|\frac{\partial u}{\partial x_{i}}\right|^{p_{i}(x)-1} \chi_{F}\right) \leq K, \quad \forall i=1, \ldots, N,
$$

where $F=\{h<|u| \leq h+k\}, h>0, k>0$.

Proof. Let $\varphi=T_{h}(u)$ as test function in the entropy inequality (36). We get

$$
\begin{gathered}
\sum_{i=1}^{N} \int_{\Omega} a_{i}\left(x, \frac{\partial u}{\partial x_{i}}\right) \frac{\partial}{\partial x_{i}} T_{k}\left(u-T_{h}(u)\right) d x \\
\quad+\int_{\Omega} b(u) T_{k}\left(u-T_{h}(u)\right) d x \\
\quad+\int_{\partial \Omega} \gamma(u) T_{k}\left(u-T_{h}(u)\right) d \sigma \\
\leq \int_{\Omega} f T_{k}\left(u-T_{h}(u)\right) d x .
\end{gathered}
$$

Thus,

$$
\sum_{i=1}^{N} \int_{\{h<|u| \leq h+k\}} a_{i}\left(x, \frac{\partial u}{\partial x_{i}}\right) \frac{\partial u}{\partial x_{i}} d x \leq k\|f\|_{1}
$$

and using (7), we have

$$
\int_{F}\left|\frac{\partial u}{\partial x_{i}}\right|^{p_{i}(x)} d x \leq \frac{k}{C_{3}}\|f\|_{1}, \quad \forall i=1, \ldots, N .
$$

Consequently,

$$
\rho_{p_{i}^{\prime}(\cdot)}\left(\left|\frac{\partial u}{\partial x_{i}}\right|^{p_{i}(x)-1} \chi_{F}\right) \leq K, \quad \forall i=1, \ldots, N
$$

Step 2 (uniqueness of entropy solution). Let $h>0$ and $u, v$ be two entropy solutions of (1). We write the entropy inequality corresponding to the solution $u$, with $T_{h}(v)$ as test function, and to the solution $v$, with $T_{h}(u)$ as test function to get

$$
\begin{aligned}
& \sum_{i=1}^{N} \int_{\Omega} a_{i}\left(x, \frac{\partial u}{\partial x_{i}}\right) \frac{\partial}{\partial x_{i}} T_{k}\left(u-T_{h}(v)\right) d x \\
& \quad+\int_{\Omega} b(u) T_{k}\left(u-T_{h}(v)\right) d x \\
& \quad+\int_{\partial \Omega} \gamma(u) T_{k}\left(u-T_{h}(v)\right) d \sigma \\
& \quad \leq \int_{\Omega} f T_{k}\left(u-T_{h}(v)\right) d x, \\
& \sum_{i=1}^{N} \int_{\Omega} a_{i}\left(x, \frac{\partial v}{\partial x_{i}}\right) \frac{\partial}{\partial x_{i}} T_{k}\left(v-T_{h}(u)\right) d x \\
& \quad+\int_{\Omega} b(v) T_{k}\left(v-T_{h}(u)\right) d x \\
& \quad+\int_{\partial \Omega} \gamma(v) T_{k}\left(v-T_{h}(u)\right) d \sigma \\
& \quad \leq \int_{\Omega} f T_{k}\left(v-T_{h}(u)\right) d x .
\end{aligned}
$$

Upon addition, we get

$$
\begin{aligned}
& \sum_{i=1}^{N} \int_{\Omega} a_{i}\left(x, \frac{\partial u}{\partial x_{i}}\right) \frac{\partial}{\partial x_{i}} T_{k}\left(u-T_{h}(v)\right) d x \\
& \quad+\sum_{i=1}^{N} \int_{\Omega} a_{i}\left(x, \frac{\partial v}{\partial x_{i}}\right) \frac{\partial}{\partial x_{i}} T_{k}\left(v-T_{h}(u)\right) d x \\
& \quad+\int_{\Omega} b(u) T_{k}\left(u-T_{h}(v)\right) d x \\
& \quad+\int_{\Omega} b(v) T_{k}\left(v-T_{h}(u)\right) d x \\
& \quad+\int_{\partial \Omega} \gamma(u) T_{k}\left(u-T_{h}(v)\right) d \sigma \\
& \quad+\int_{\partial \Omega} \gamma(v) T_{k}\left(v-T_{h}(u)\right) d \sigma \\
& \leq \int_{\Omega} f\left[T_{k}\left(u-T_{h}(v)\right)+T_{k}\left(v-T_{h}(u)\right)\right] d x .
\end{aligned}
$$


Define the following sets:

$$
\begin{aligned}
& E_{1}\{|u-v| \leq k ;|v| \leq h\} ; \\
& E_{2} E_{1} \cap\{|u| \leq h\}, \\
& E_{3} E_{1} \cap\{|u|>h\} .
\end{aligned}
$$

We start with the first integral in (135). We have

$$
\begin{aligned}
& \sum_{i=1}^{N} \int_{\left\{\left|u-T_{h}(v)\right| \leq k\right\}} a_{i}\left(x, \frac{\partial u}{\partial x_{i}}\right) \frac{\partial}{\partial x_{i}} T_{k}\left(u-T_{h}(v)\right) d x \\
& =\sum_{i=1}^{N} \int_{\left\{\left|u-T_{h}(v)\right| \leq k\right\} \cap\{|v| \leq h\}} a_{i}(x, \\
& \left.\frac{\partial u}{\partial x_{i}}\right) \frac{\partial}{\partial x_{i}} T_{k}\left(u-T_{h}(v)\right) d x \\
& +\sum_{i=1}^{N} \int_{\left\{\left|u-T_{h}(v)\right| \leq k\right\} \cap\{|v|>h\}} a_{i}(x, \\
& \left.\frac{\partial u}{\partial x_{i}}\right) \frac{\partial}{\partial x_{i}} T_{k}\left(u-T_{h}(v)\right) d x \\
& =\sum_{i=1}^{N} \int_{\{|u-v| \leq k\} \cap\{|v| \leq h\}} a_{i}\left(x, \frac{\partial u}{\partial x_{i}}\right) \frac{\partial(u-v)}{\partial x_{i}} d x \\
& +\sum_{i=1}^{N} \int_{\{|u-h \operatorname{sign}(v)| \leq k\} \cap\{|v|>h\}} a_{i}\left(x, \frac{\partial u}{\partial x_{i}}\right) \frac{\partial u}{\partial x_{i}} d x \\
& \geq \sum_{i=1}^{N} \int_{E_{1}} a_{i}\left(x, \frac{\partial u}{\partial x_{i}}\right) \frac{\partial}{\partial x_{i}}(u-v) d x \geq \sum_{i=1}^{N} \int_{E_{2}} a_{i}(x, \\
& \left.\frac{\partial u}{\partial x_{i}}\right) \frac{\partial}{\partial x_{i}}(u-v) d x \\
& +\sum_{i=1}^{N} \int_{E_{3}} a_{i}\left(x, \frac{\partial u}{\partial x_{i}}\right) \frac{\partial}{\partial x_{i}}(u-v) d x .
\end{aligned}
$$

Then, we obtain

$$
\begin{gathered}
\sum_{i=1}^{N} \int_{\left\{\left|u-T_{h}(v)\right| \leq k\right\}} a_{i}\left(x, \frac{\partial u}{\partial x_{i}}\right) \frac{\partial}{\partial x_{i}} T_{k}\left(u-T_{h}(v)\right) d x \\
\geq \sum_{i=1}^{N} \int_{E_{2}} a_{i}\left(x, \frac{\partial u}{\partial x_{i}}\right) \frac{\partial}{\partial x_{i}}(u-v) d x \\
\quad-\sum_{i=1}^{N} \int_{E_{3}} a_{i}\left(x, \frac{\partial u}{\partial x_{i}}\right) \frac{\partial v}{\partial x_{i}} d x .
\end{gathered}
$$

According to (5) and the Hölder type inequality, we have

$$
\begin{aligned}
& \left|\sum_{i=1}^{N} \int_{E_{3}} a_{i}\left(x, \frac{\partial u}{\partial x_{i}}\right) \frac{\partial v}{\partial x_{i}} d x\right| \\
& \quad \leq C_{1} \sum_{i=1}^{N} \int_{E_{3}}\left(j_{i}(x)+\left|\frac{\partial u}{\partial x_{i}}\right|^{p_{i}(x)-1}\right)\left|\frac{\partial v}{\partial x_{i}}\right| d x \\
& \leq C_{1} \sum_{i=1}^{N}\left(\left|j_{i}\right|_{p_{i}^{\prime}(\cdot)}+\left.\left.|| \frac{\partial u}{\partial x_{i}}\right|^{p_{i}(x)-1}\right|_{p_{i}^{\prime}(\cdot),\{h<|u| \leq h+k\}}\right) \\
& \cdot\left|\frac{\partial v}{\partial x_{i}}\right|_{p_{i}(\cdot),\{h-k<|v| \leq h\}},
\end{aligned}
$$

where

$$
\begin{gathered}
\|\left.\left.\frac{\partial u}{\partial x_{i}}\right|^{p_{i}(x)-1}\right|_{p_{i}^{\prime}(\cdot),\{h<|u| \leq h+k\}} \\
=\left\|\left|\frac{\partial u}{\partial x_{i}}\right|^{p_{i}(x)-1}\right\|_{L^{p_{i}^{\prime}(\cdot)}(\{h<|u| \leq h+k\})} .
\end{gathered}
$$

Thanks to relation (18) and Lemma 16, the quantity $\left(\left|j_{i}\right|_{p_{i}^{\prime}(\cdot)}+\right.$ $\left.\| \partial u /\left.\left.\partial x_{i}\right|^{p_{i}(x)-1}\right|_{p_{i}^{\prime}(\cdot),\{h<|u| \leq h+k\}}\right)$ is finite for all $i=1, \ldots, N$.

According to Lemma 15, the quantity $|\partial v|$ $\left.\partial x_{i}\right|_{p_{i}(\cdot),\{h-k<|v| \leq h\}}$ converges to zero as $h$ goes to infinity. Consequently, the last integral of (138) converges to zero as $h$ goes to infinity. Then,

$$
\begin{gathered}
\sum_{i=1}^{N} \int_{\left\{\left|u-T_{h}(v)\right| \leq k\right\}} a_{i}\left(x, \frac{\partial u}{\partial x_{i}}\right) \frac{\partial}{\partial x_{i}} T_{k}\left(u-T_{h}(v)\right) d x \\
\geq I_{h}+\sum_{i=1}^{N} \int_{E_{2}} a_{i}\left(x, \frac{\partial u}{\partial x_{i}}\right) \frac{\partial}{\partial x_{i}}(u-v) d x,
\end{gathered}
$$

with $\lim _{h \rightarrow+\infty} I_{h}=0$.

We may adopt the same procedure to treat the second term in (135) to obtain

$$
\begin{gathered}
\sum_{i=1}^{N} \int_{\left\{\left|v-T_{h}(u)\right| \leq k\right\}} a_{i}\left(x, \frac{\partial v}{\partial x_{i}}\right) \frac{\partial}{\partial x_{i}} T_{k}\left(v-T_{h}(u)\right) d x \\
\geq J_{h}-\sum_{i=1}^{N} \int_{E_{2}} a_{i}\left(x, \frac{\partial v}{\partial x_{i}}\right) \frac{\partial}{\partial x_{i}}(u-v) d x,
\end{gathered}
$$

with $\lim _{h \rightarrow+\infty} J_{h}=0$.

For the other terms in the left-hand side of (135), we denote

$$
\begin{aligned}
K_{h}= & \int_{\Omega} b(u) T_{k}\left(u-T_{h}(v)\right) d x \\
& +\int_{\Omega} b(v) T_{k}\left(v-T_{h}(u)\right) d x, \\
L_{h}= & \int_{\partial \Omega} \gamma(u) T_{k}\left(u-T_{h}(v)\right) d \sigma \\
& +\int_{\partial \Omega} \gamma(v) T_{k}\left(v-T_{h}(u)\right) d \sigma .
\end{aligned}
$$


We have

$$
\begin{gathered}
b(u) T_{k}\left(u-T_{h}(v)\right) \longrightarrow b(u) T_{k}(u-v) \\
\text { a.e. in } \Omega \text { since } h \longrightarrow+\infty, \\
\left|b(u) T_{k}\left(u-T_{h}(v)\right)\right| \leq k|b(u)| \in L^{1}(\Omega) .
\end{gathered}
$$

Then, by the Lebesgue dominated convergence theorem, we obtain

$$
\begin{aligned}
& \lim _{h \rightarrow+\infty} \int_{\Omega} b(u) T_{k}\left(u-T_{h}(v)\right) d x \\
& \quad=\int_{\Omega} b(u) T_{k}(u-v) d x, \\
& \lim _{h \rightarrow+\infty} \int_{\Omega} b(v) T_{k}\left(v-T_{h}(u)\right) d x \\
& =\int_{\Omega} b(v) T_{k}(v-u) d x .
\end{aligned}
$$

Then,

$$
\lim _{h \rightarrow+\infty} K_{h}=\int_{\Omega}(b(u)-b(v)) T_{k}(u-v) d x .
$$

In the same way, we get

$$
\lim _{h \rightarrow+\infty} L_{h}=\int_{\partial \Omega}(\gamma(u)-\gamma(v)) T_{k}(u-v) d \sigma .
$$

Now, consider the right-hand side of inequality (135). We have

$$
\begin{aligned}
& \lim _{h \rightarrow+\infty} f\left(T_{k}\left(u-T_{h}(v)\right)+T_{k}\left(v-T_{h}(u)\right)\right)=0 \\
& \text { a.e. in } \Omega, \\
& \left|f(x)\left(T_{k}\left(u-T_{h}(v)\right)+T_{k}\left(v-T_{h}(u)\right)\right)\right| \leq 2 k|f| \\
& \quad \in L^{1}(\Omega) .
\end{aligned}
$$

By the Lebesgue dominated convergence theorem, we obtain

$$
\begin{aligned}
& \lim _{h \rightarrow+\infty} \int_{\Omega} f(x)\left(T_{k}\left(u-T_{h}(v)\right)+T_{k}\left(v-T_{h}(u)\right)\right) d x \\
& \quad=0 .
\end{aligned}
$$

After passing to the limit as $h$ goes to $+\infty$ in (135), we get

$$
\begin{aligned}
& \sum_{i=1}^{N} \int_{\{|u-v| \leq k\}}\left(a_{i}\left(x, \frac{\partial u}{\partial x_{i}}\right)\right. \\
& \left.\quad-a_{i}\left(x, \frac{\partial v}{\partial x_{i}}\right)\right) \frac{\partial}{\partial x_{i}}(u-v) d x \\
& \quad+\int_{\Omega}(b(u)-b(v)) T_{k}(u-v) d x+\int_{\partial \Omega}(\gamma(u) \\
& \quad-\gamma(v)) T_{k}(u-v) d \sigma \leq 0 .
\end{aligned}
$$

Since $b, \gamma$, and $a_{i}(x, \cdot)$ are monotone, then

$$
\begin{aligned}
& \int_{\Omega}(b(u)-b(v)) T_{k}(u-v) d x=0 \\
& \int_{\partial \Omega}(\gamma(u)-\gamma(v)) T_{k}(u-v) d \sigma=0 \\
& \int_{\{|u-v| \leq k\}} \sum_{i=1}^{N}\left(a_{i}\left(x, \frac{\partial u}{\partial x_{i}}\right)\right. \\
& \left.-a_{i}\left(x, \frac{\partial v}{\partial x_{i}}\right)\right) \frac{\partial}{\partial x_{i}}(u-v) d x \\
& =0 .
\end{aligned}
$$

According to (6), we deduce from (153) that

$$
u-v=c \text { a.e. } x \in \Omega \text {, where } c \text { is a real constant. }
$$

For $x$ fixed in $\partial \Omega, s \mapsto|s|^{p(x)-2} s$ is nondecreasing and vanishes at 0 . Then,

$$
\begin{aligned}
&(\gamma(u(x))-\gamma(v(x))) T_{k}(u(x)-v(x)) \geq 0, \\
& \forall x \in \partial \Omega, \forall k>0 .
\end{aligned}
$$

Now, by inequality above and (152), we deduce that for all $k \in$ $\mathbb{N}^{*}$ there exists $C_{k} \subset \partial \Omega$ with meas $\left(C_{k}\right)=0$ such that, for all $x \in \partial \Omega \backslash C_{k}$,

$$
(\gamma(u(x))-\gamma(v(x))) T_{k}(u(x)-v(x))=0 .
$$

Therefore,

$$
\begin{aligned}
(\gamma(u(x))-\gamma(v(x)))(u(x)-v(x)) & =0, \\
\forall x & \in \partial \Omega \backslash \bigcup_{k \in \mathbb{N}^{*}} C_{k} .
\end{aligned}
$$

As $p^{-}>1$, the following relation is true for any $\xi, \eta \in \mathbb{R}, \xi \neq \eta$ (cf. [14]):

$$
\left(|\xi|^{p(x)-2} \xi-|\eta|^{p(x)-2} \eta\right)(\xi-\eta)>0 .
$$

From inequality above and (157), we get

$$
u-v=0 \text { a.e. on } \partial \Omega \text {. }
$$

Finally as

$$
\begin{array}{ll}
u-v=c & \text { a.e. in } \Omega, \\
u-v=0 & \text { a.e. on } \partial \Omega,
\end{array}
$$

it follows that

$$
u=v \quad \text { a.e. in } \Omega \text {. }
$$

That completes the proof of Theorem 12 .

\section{Conflict of Interests}

The authors declare that there is no conflict of interests regarding the publication of this paper. 


\section{References}

[1] B. K. Bonzi, S. Ouaro, and F. D. Y. Zongo, "Entropy solutions to nonlinear elliptic anisotropic problem with Robin boundary condtion," Le Matematiche, vol. 68, no. 2, pp. 53-76, 2013.

[2] M. Mihǎilescu, P. Pucci, and V. Rădulescu, "Eigenvalue problems for anisotropic quasilinear elliptic equations with variable exponent," Journal of Mathematical Analysis and Applications, vol. 340, no. 1, pp. 687-698, 2008.

[3] M.-M. Boureanu and V. D. Radulescu, "Anisotropic Neumann problems in Sobolev spaces with variable exponent," Nonlinear Analysis, Theory, Methods and Applications, vol. 75, no. 12, pp. 4471-4482, 2012.

[4] B. Kon, S. Ouaro, and S. Traor, "Weak solutions for anisotropic nonlinear elliptic equations with variable exponents," Electronic Journal of Differential Equations, vol. 2009, no. 144, pp. 1-11, 2009.

[5] S. Ouaro, "Well-posedness results for anisotropic nonlinear elliptic equations with variable exponent and $L^{1}$-data," Cubo Journal, vol. 12, no. 1, pp. 133-148, 2010.

[6] P. Bénilan, L. Boccardo, T. Gallouët, R. Gariepy, M. Pierre, and J. L. Vazguez, "An $L^{1}$-theory of existence and uniqueness of solutions of nonlinear elliptic equations," Annali della Scuola Normale Superiore di Pisa. Classe di Scienze 4, vol. 22, no. 2, pp. 241-273, 1995.

[7] B. K. Bonzi and S. Ouaro, "Entropy solutions for a doubly nonlinear elliptic problem with variable exponent," Journal of Mathematical Analysis and Applications, vol. 370, no. 2, pp. 392405, 2010.

[8] R. E. Showalter, Monotone Operators in Banach Space and Nonlinear Partial Differential Equations, vol. 49 of Mathematical Surveys and Monographs, American Mathematical Society, 1991.

[9] X. Fan, "Anisotropic variable exponent Sobolev spaces and $\vec{p}($.$) -$ Laplacien equations," Complex Variables and Elliptic Equations, vol. 55, pp. 1-20, 2010.

[10] X. Fan and D. Zhao, "On the spaces $L^{p(x)}(\Omega)$ and $W^{1, p(x)}(\Omega)$," Journal of Mathematical Analysis and Applications, vol. 263, pp. 424-446, 2001.

[11] O. Kovacik and Z. J. Rakosnik, "On spaces $L^{p(x)}$ and $W^{1, p(x), "}$ Czechoslovak Mathematical Journal, vol. 41, pp. 592-618, 1991.

[12] M. Troisi, "Theoremi di inclusione per spazi di Sobolev non isotropi," Ricerche di Matematica, vol. 18, pp. 3-24, 1969.

[13] B. K. Bonzi, S. Ouaro, and F. D. Y. Zongo, "Entropy solutions for nonlinear elliptic anisotropic homogeneous Neumann problem," International Journal of Differential Equations, vol. 2013, Article ID 476781, 14 pages, 2013.

[14] X.-L. Fan and Q.-H. Zhang, "Existence of solutions for $p(x)$ Laplacian Dirichlet problem," Nonlinear Analysis: Theory, Methods \& Applications, vol. 52, no. 8, pp. 1843-1852, 2003. 


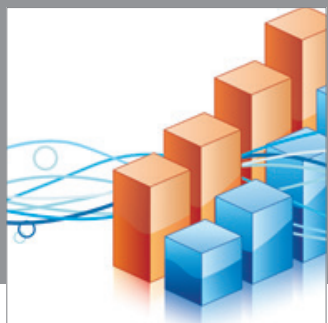

Advances in

Operations Research

mansans

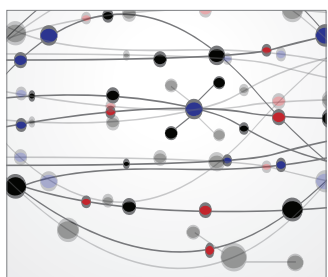

The Scientific World Journal
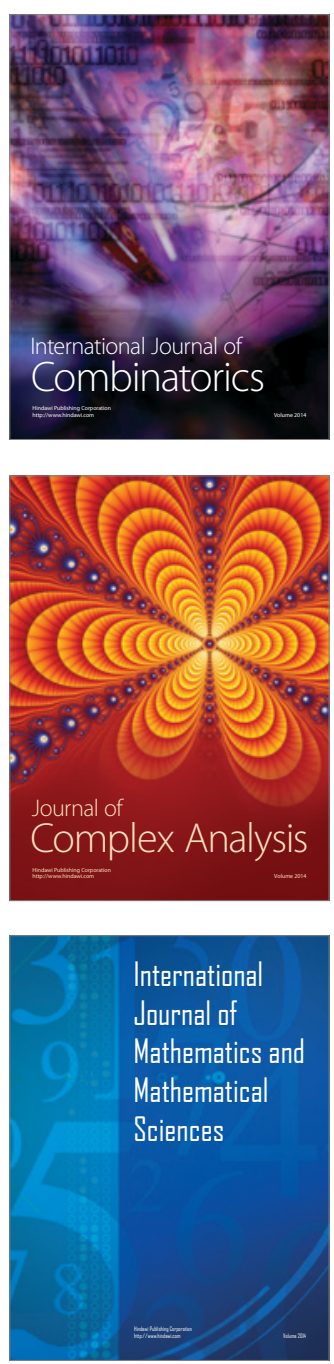
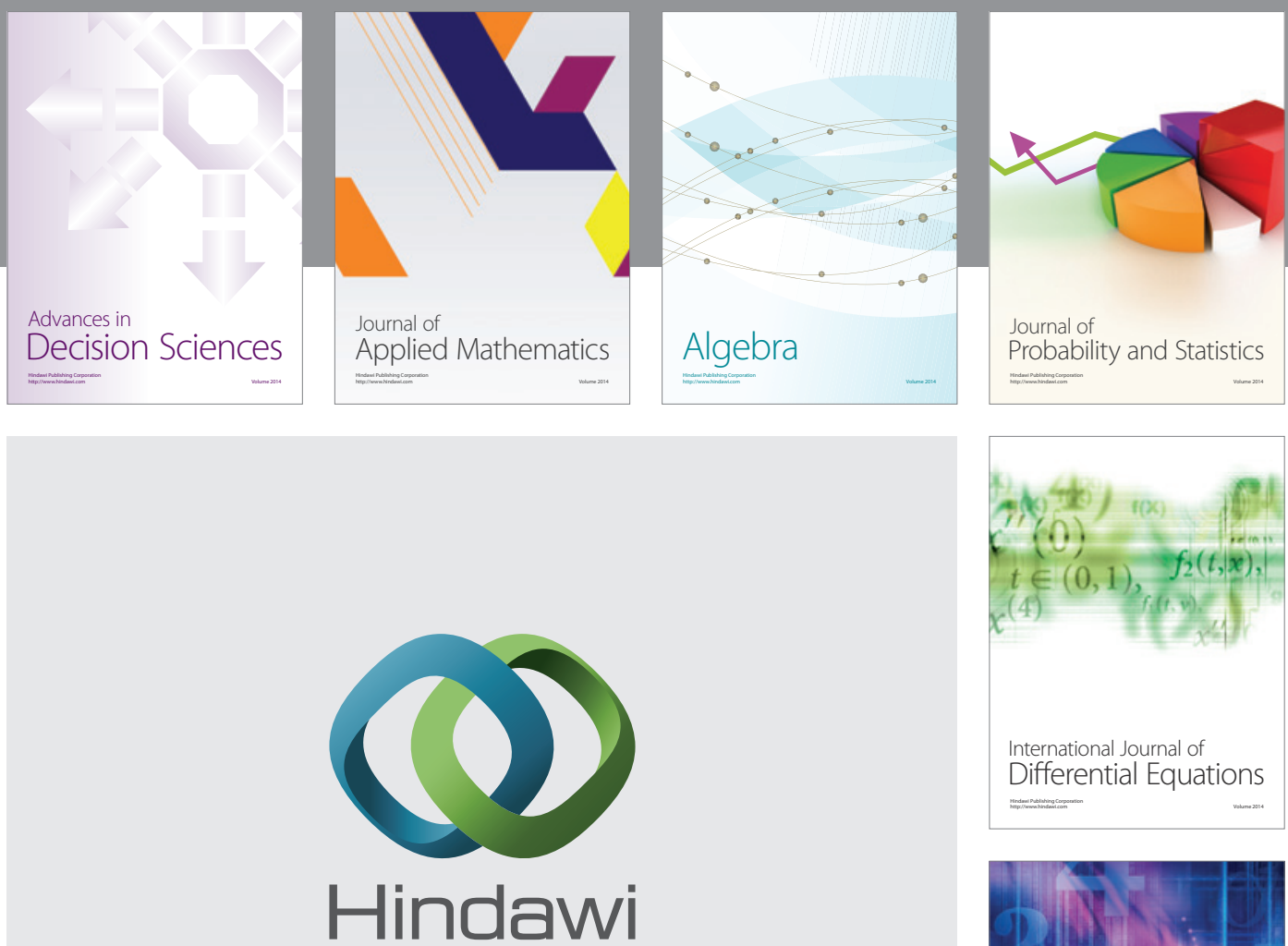

Submit your manuscripts at http://www.hindawi.com
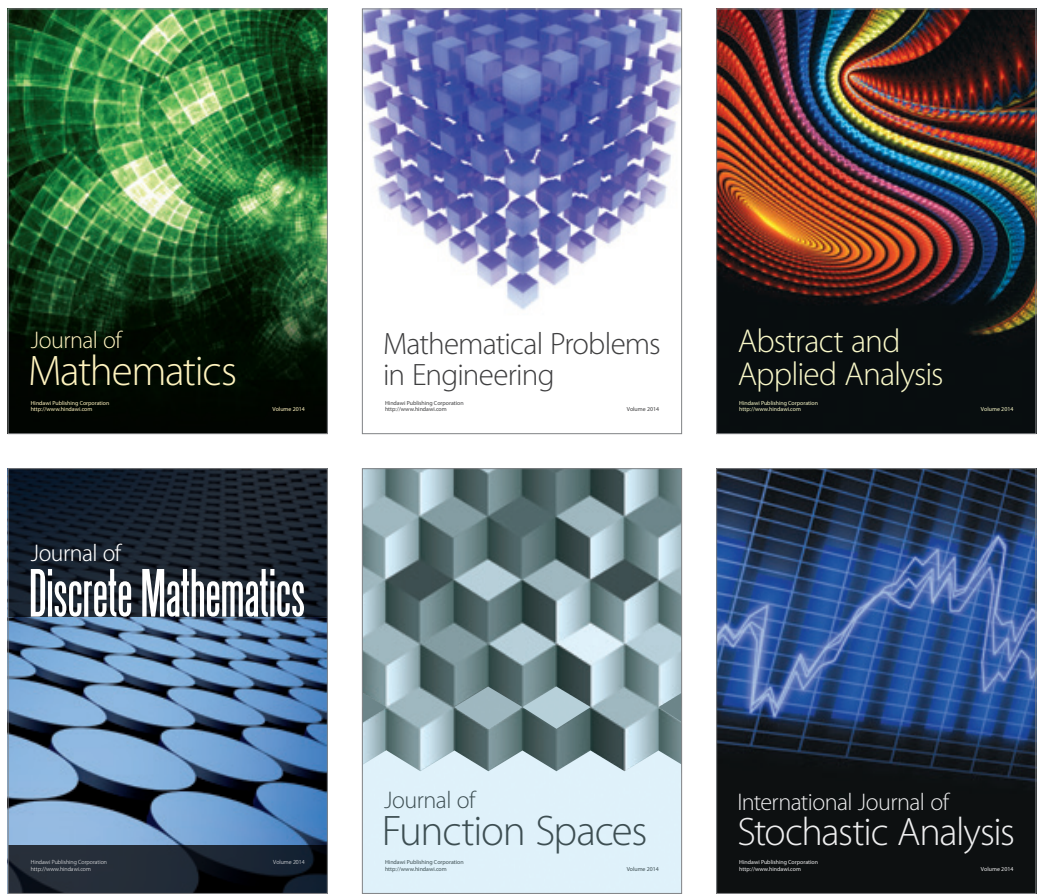

Journal of

Function Spaces

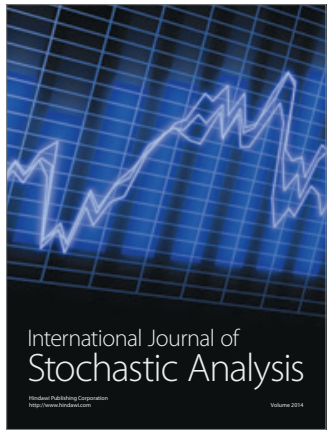

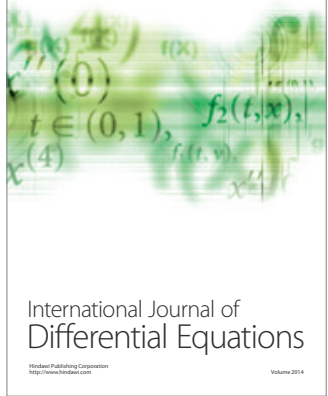
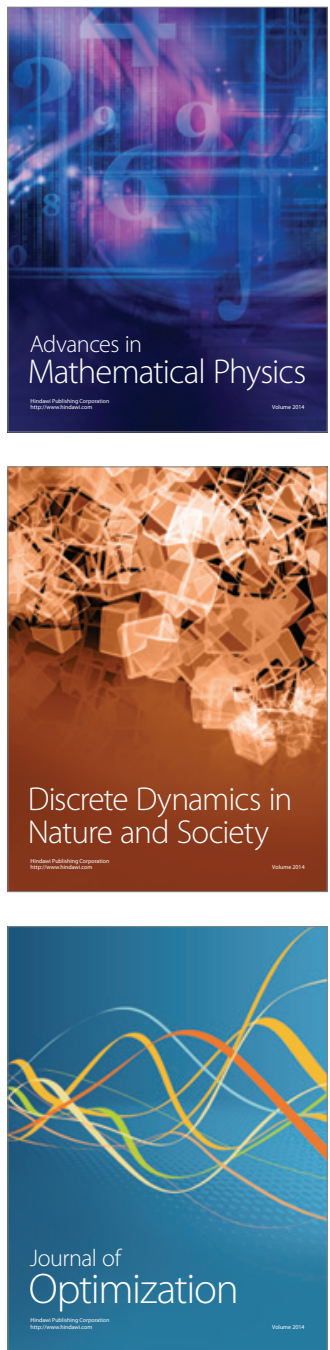